\title{
Relationships between radium and radon occurrence and hydrochemistry in fresh groundwater from fractured crystalline rocks, North Carolina (USA)
}

\author{
David S. Vinson*, Avner Vengosh, Daniella Hirschfeld, Gary S. Dwyer \\ Division of Earth and Ocean Sciences, Nicholas School of the Environment, Box 90227, Duke University, Durham, NC 27708, United States
}

\section{A R T I C L E I N F O}

Article history:

Accepted 21 October 2008

\section{Keywords:}

Radium

Radon

Uranium

Redox

Fractured crystalline rock

\begin{abstract}
A B S T R A C T
Naturally-occurring radionuclides (uranium, radium, and radon), major dissolved constituents, and trace elements were investigated in fresh groundwater in 117 wells in fractured crystalline rocks from the Piedmont region (North Carolina, USA). Chemical variations show a general transition between two water types: (1) slightly acidic ( $\mathrm{pH}$ 5.0-6.0), oxic, low-total dissolved solids (TDS) waters, and (2) near neutral, oxic to anoxic, higher-TDS waters. The uranium, radium, and radon levels in groundwater associated with granite (Rolesville Granite) are systematically higher than other rock types (gneiss, metasedimentary, and metavolcanic rocks). Water chemistry plays a secondary role on radium and radon distributions as the ${ }^{222} \mathrm{Rn} /{ }^{226} \mathrm{Ra}$ activity ratio is correlated with redox-sensitive solutes such as dissolved oxygen and Mn concentrations, as well as overall dissolved solids content including major divalent cations and Ba. Since ${ }^{224} \mathrm{Ra} /{ }^{228} \mathrm{Ra}$ activity ratios in groundwater are close to 1 , we suggest that mobilization of Ra and $\mathrm{Rn}$ is controlled by alpha recoil processes from parent nuclides on fracture surfaces, ruling out Ra sources from mineral dissolution or significant long-distance Ra transport. Alpha recoil is balanced by Ra adsorption that is influenced by redox conditions and/or ion concentrations, resulting in an approximately one order of magnitude decrease $(\sim 20,000$ to $\sim 2000)$ in the apparent Ra distribution coefficient between oxygensaturated and anoxic conditions and also across the range of dissolved ion concentrations (up to $\sim 7 \mathrm{mM}$ ). Thus, the $U$ and Th content of rocks is the primary control on observed Ra and Rn activities in groundwater in fractured crystalline rocks, and in addition, linked dissolved solids concentrations and redox conditions impart a secondary control.
\end{abstract}

(c) 2008 Elsevier B.V. All rights reserved.

\section{Introduction}

Groundwater sources of drinking water may contain naturallyoccurring radionuclides from the uranium-238, uranium-235, and thorium-232 series, especially uranium (U), radium (Ra), and radon $(\mathrm{Rn})$, all of which are the basis of current $(\mathrm{U}, \mathrm{Ra})$ or proposed $(\mathrm{Rn})$ drinking water standards of the U.S. Environmental Protection Agency (EPA). Uranium is a source of kidney toxicity as well as alpha radiation (EPA, 2000). Radium is a bone-seeking form of natural radioactivity when consumed in drinking water (Mays et al., 1985). Radon is a radioactive gas for which both ingestion and inhalation present health risks, although inhalation may provide about one order of magnitude larger exposure (National Research Council, 1999). U, Ra, and Rn may co-occur in high concentrations in the case of mineralized areas (e.g. Asikainen and Kahlos, 1979), but in most cases, strong disequilibrium exists among $\mathrm{U}, \mathrm{Ra}$, and $\mathrm{Rn}$ in the groundwater of crystalline rocks. Disequilibrium is observed because of geochemical conditions that preferentially mobilize U and/or Ra as well as the inert nature of Rn. For

\footnotetext{
* Corresponding author. Tel.: +1 9196819339.

E-mail address: dsv3@duke.edu (D.S. Vinson).
}

example, waters may be high in U but low in other nuclides (Asikainen, 1981b); orders of magnitude higher in Rn than in Ra (King et al., 1982; Wanty et al., 1991); or high in Ra but relatively low in U in deep, anoxic waters (Andrews et al., 1989; Gascoyne, 1989).

In addition to being of significance as natural drinking water contaminants, Ra isotopes are useful tools to evaluate the behavior and reactivity of radionuclides in aquifer systems. This is due to (1) the large range of half-lives ( $3.7 \mathrm{~d}$ for ${ }^{224} \mathrm{Ra}, 11.4 \mathrm{~d}$ for ${ }^{223} \mathrm{Ra}, 5.8$ a for ${ }^{228} \mathrm{Ra}$, and 1600 a for ${ }^{226} \mathrm{Ra}$ ); and (2) the highly reactive nature of Ra, especially used in conjunction with the inert nature of radon-222 (half-life $3.8 \mathrm{~d}$ ), the daughter of ${ }^{226} \mathrm{Ra}$. In fresh waters, the relationships between Ra isotopes and between $\mathrm{Ra}$ and $\mathrm{Rn}$ suggest that Ra is generated primarily by alpha recoil from the decay of its Th parents on the aquifer solids, rather than by weathering processes, and that transport of Ra along a flowpath is retarded several orders of magnitude relative to conservative solutes (Krishnaswami et al., 1982; Dickson, 1990; Porcelli, 2008). Thus, $\mathrm{Ra}$ isotope ratios and $\mathrm{Rn} / \mathrm{Ra}$ ratios can indicate the balance between physical processes (alpha recoil) and geochemical processes such as Ra adsorption.

Radium mobility or adsorption effectiveness is understood to vary with chemical parameters including $\mathrm{pH}$ (Cecil et al., 1987; Dickson and Herczeg, 1992; Bolton, 2000; Szabo et al., 2005), salinity (Kraemer and 
Reid, 1984; Sturchio et al., 2001; Wood et al., 2004), reduced conditions (Szabo and Zapecza, 1987; Herczeg et al., 1988), supersaturation with respect to barite (Gilkeson et al., 1984; Grundl and Cape, 2006), microbial sulfate reduction affecting barite stability (Phillips et al., 2001; Martin et al., 2003), and microbial Fe oxide reduction (Landa et al., 1991). Ra adsorption is a rapid influence in freshwater at nearneutral pH (Krishnaswami et al., 1982), but the effects of redox processes and relatively low ion concentrations on adsorption and desorption are not well constrained in existing field investigations. In addition, many previous studies of Ra in fresh waters have not included detailed characterization of redox conditions or analysis of short-lived $\mathrm{Ra}$ isotopes with which to apply the full range of Ra half-lives to geochemical conditions. In this research, we focus chemical and Ra isotope investigation on fresh, near-neutral, bicarbonate-dominated groundwaters from fractured crystalline rocks in North Carolina (USA). The objectives of the study are to evaluate mechanisms of Ra and Rn mobilization in shallow groundwater and to elucidate the hydrochemical influences, if any, on observed Ra and Rn activities under these conditions.

\section{Methods}

\subsection{Sampling procedures}

117 private wells were sampled in Wake County, North Carolina, clustered into six groups (Fig. 1): (1a) and (1b) are a complexly mapped area mostly composed of late Proterozoic to early Paleozoic metasedimentary and metavolcanic rocks (felsic gneiss, felsic schist, phyllite, meta-tuff, and scattered ultramafic rocks) and associated felsic intrusions including quartz diorites (Stoddard et al., 1991; Clark et al., 2004); (2) is primarily underlain by the early Paleozoic Raleigh Gneiss, composed of sedimentary and granitic components (Stoddard et al., 1991; Clark et al., 2004); and (3a), (3b), and (3c) are underlain by the late Paleozoic Rolesville granitic pluton, a large, complex unit composed of monzogranite, granite, granodiorite and pegmatite in places (Speer, 1994; Clark et al., 2004). The study area is also intruded by Mesozoic dikes of mafic composition (Clark et al., 2004).

Samples were collected at wellhead taps where available, or at outdoor taps after ensuring that samples were not affected by chemical

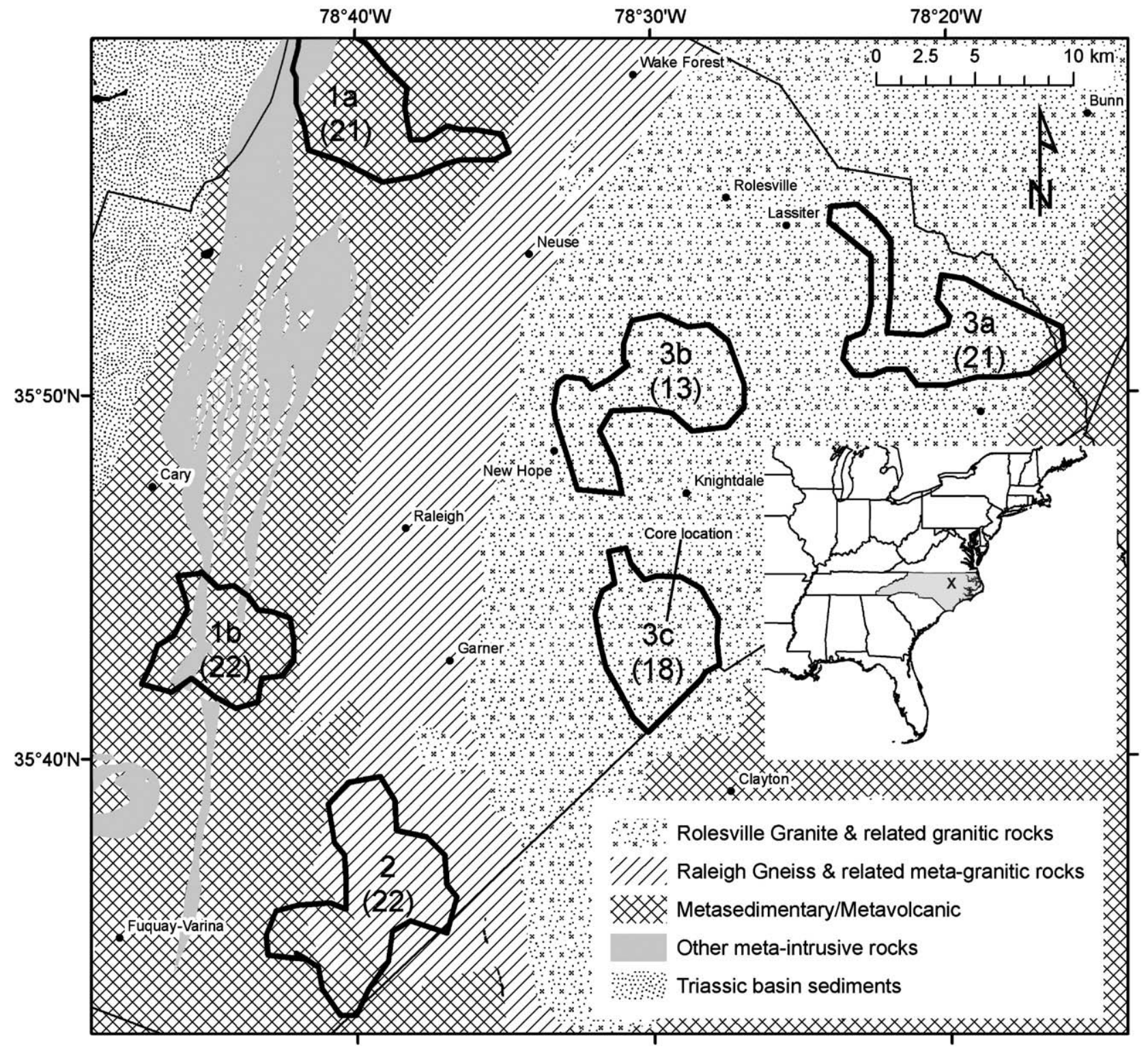

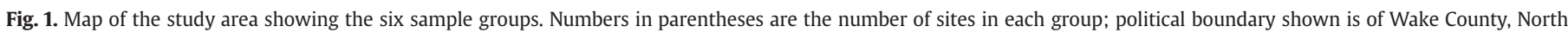
Carolina. Geological data based on Clark et al. (2004); data for location map obtained from www.nationalatlas.gov. 
treatment systems. Dissolved oxygen (DO) and pH were measured from a continuous flow at the bottom of a polyethylene bucket using YSI DO200 and pH100 meters, respectively. Also from this continuous flow, a radon sample was collected into a submerged glass vial, and an unfiltered alkalinity sample was collected into a polyethylene bottle. Trace metal/cation samples were filtered in the field directly into new, high-purity acid-washed polyethylene bottles containing high-purity $\mathrm{HNO}_{3}$ using syringe-tip $0.45 \mu \mathrm{m}$ filters. Anion samples were similarly field-filtered. In the lab, Ra isotopes were concentrated from $52 \mathrm{~L}$ samples onto $10 \mathrm{~g}$ bundles of Mn oxide-coated acrylic fibers (Moore and Reid, 1973; Reid et al., 1979) at a flow rate of $<1 \mathrm{~L} \mathrm{~min}^{-1}$. 19 of the 117 samples were concentrated onto one column of Mn oxide fibers and the remaining 98 samples were concentrated onto two sequential (A and B) columns (e.g. Luo et al., 2000). Radon-222 samples were shipped overnight to a commercial laboratory for determination by liquid scintillation counting and are decay-corrected to the time of collection.

\subsection{Radium isotope analysis}

Analysis of ${ }^{224} \mathrm{Ra}$ was carried out as soon as possible after concentration onto Mn oxide fibers by alpha counting using a delayed coincidence counter (Moore and Arnold, 1996). For fibers with less than 20 total alpha counts per minute at time of analysis, ${ }^{224} \mathrm{Ra}$ was quantified using the ${ }^{220} \mathrm{Rn}$ channel, corrected for chance coincidence events. For fibers with greater than 20 counts per minute, ${ }^{224} \mathrm{Ra}$ was quantified by total counts, corrected for activity attributable to ${ }^{223} \mathrm{Ra}$ (Garcia-Solsona et al., 2008) and for ${ }^{224} \mathrm{Ra}-{ }^{220} \mathrm{Rn}$ disequilibrium during short run times. ${ }^{224} \mathrm{Ra}$ was calibrated by an old, equilibrated ${ }^{232} \mathrm{Th}$ solution that was transferred onto Mn oxide fibers. ${ }^{224} \mathrm{Ra}$ was decaycorrected to the time of collection and for ${ }^{224} \mathrm{Ra}$ supported by small amounts of ${ }^{228} \mathrm{Th}$ adsorbed onto the fibers after 3-6 weeks. ${ }^{226} \mathrm{Ra}$ analysis was performed by radon counting after 20 days of incubation in a sealed, evacuated glass column on a Durridge RAD7 alpha counting instrument. ${ }^{226} \mathrm{Ra}$ standards are from a NIST solution transferred onto Mn oxide fibers. ${ }^{228} \mathrm{Ra}$ analysis was performed on a Canberra broadenergy Ge gamma spectrometer. In order to produce a more efficient (e.g. Buesseler et al., 1995) and homogeneous (e.g. Herranz et al., 2006) counting geometry, ${ }^{228} \mathrm{Ra}$ analysis was performed on fibers of the $\mathrm{A}$ column that had been compressed into a disk geometry $65 \mathrm{~mm}$ in diameter and $\sim 5 \mathrm{~mm}$ thick in a metal canister. ${ }^{228}$ Ra was quantified from a weighted average of counts from the $338 \mathrm{keV}$ and $911 \mathrm{keV}$ peaks of ${ }^{228} \mathrm{Ac}$ and corrected for the partitioning of Ra between the A and B columns using relative ${ }^{224} \mathrm{Ra}$ activities. The standard for gamma spectrometric analysis was U-Th ore standard DL-1a (Canadian Certified Reference Materials Project), loaded to resemble the geometry of the compressed fibers. For radium isotope analyses, propagation of error equations were applied to background subtraction, delayed coincidence counter corrections, and addition of radium activities from multiple columns using the methods of Ivanovich and Murray (1992) and GarciaSolsona et al. (2008). Reported $2 \sigma$ counting error (Table 4 ) approximates precision determined by repeated analyses of samples and standards at comparable activities: (1) replicate analysis of a ${ }^{226} \mathrm{Ra}$ standard (equivalent to a $67 \mathrm{mBq} \mathrm{L}^{-1}$ sample) indicates relative $2 \sigma$ of $4 \%(n=8)$; (2) routine duplicate runs of ${ }^{224} \mathrm{Ra}$ standards (equivalent to a $10 \mathrm{mBq} \mathrm{L}{ }^{-1}$ sample) indicate an average difference of $9 \%$ between duplicates $(n=9$ pairs); (3) replicate analysis during one day of a low-activity sample containing $3 \mathrm{mBq} \mathrm{L}{ }^{-1}$ of ${ }^{224} \mathrm{Ra}$ indicates relative $2 \sigma$ of $12 \%$ for ${ }^{224} \mathrm{Ra}$ $(n=5)$; and (4) replicate analysis of a sample equivalent to $38 \mathrm{mBq} \mathrm{L}^{-1}$ of ${ }^{228} \mathrm{Ra}$ indicates relative $2 \sigma$ of $16 \%(n=6)$.

Rolesville Granite samples collected from cores WC-1 and WC-3 at the Raleigh Hydrogeologic Research Station (McSwain et al., in review), located within the study area (Fig. 1), were crushed to powder, sealed into completely filled plastic canisters, incubated for three weeks and analyzed for ${ }^{226} \mathrm{Ra}$ and ${ }^{228} \mathrm{Ra}$ using the $609 \mathrm{keV}$ peak of ${ }^{214} \mathrm{Bi}$ and the $911 \mathrm{keV}$ peak of ${ }^{228} \mathrm{Ac}$, respectively. These were calibrated with the DL-1a standard in the same geometry.

\subsection{Major ions and trace elements}

$\mathrm{Ca}, \mathrm{Mg}, \mathrm{Na}, \mathrm{Sr}, \mathrm{Ba}, \mathrm{Fe}, \mathrm{Mn}$, and Si concentrations were determined by direct current plasma spectrometry and $\mathrm{K}$ was determined by flame atomic absorption spectrometry, both calibrated using solutions prepared from plasma-grade single-element standards. U concentrations were determined by inductively-coupled plasma mass spectrometry calibrated with plasma-grade $\mathrm{U}$ standard. As the wells used in this study contain metal surfaces, some metal concentrations may be influenced by

Table 1

Summary of major and trace element concentrations and pH. ND indicates not detected

\begin{tabular}{|c|c|c|c|c|c|c|c|c|c|c|c|c|c|c|c|}
\hline $\begin{array}{l}\text { All data } \\
(n=117)\end{array}$ & $\frac{\mathrm{Ca}}{(\mathrm{mM})}$ & $\frac{\mathrm{Mg}}{(\mathrm{mM})}$ & $\frac{\mathrm{Na}}{(\mathrm{mM})}$ & $\frac{\mathrm{K}}{(\mathrm{mM})}$ & $\frac{\mathrm{Fe}}{(\mu \mathrm{M})}$ & $\frac{\mathrm{Mn}}{(\mu \mathrm{M})}$ & $\frac{\mathrm{Sr}}{(\mu \mathrm{M})}$ & $\frac{\mathrm{Ba}}{(\mu \mathrm{M})}$ & $\frac{\mathrm{Cl}^{-}}{(\mathrm{mM})}$ & $\frac{\mathrm{NO}_{3}^{-}}{(\mathrm{mM})}$ & $\frac{\mathrm{SO}_{4}^{2-}}{(\mathrm{mM})}$ & $\frac{\mathrm{HCO}_{3}^{-}}{(\mathrm{mM})}$ & $\frac{\mathrm{SiO}_{2}}{(\mathrm{mM})}$ & $\mathrm{pH}$ & $\frac{\mathrm{DO}}{(\mu \mathrm{M})}$ \\
\hline Mean & 0.30 & 0.13 & 0.46 & 0.05 & 4.27 & 0.85 & 0.81 & 0.23 & 0.16 & 0.097 & 0.050 & 1.10 & 0.57 & 6.36 & 129 \\
\hline Median & 0.20 & 0.11 & 0.42 & 0.05 & 0.25 & 0.15 & 0.68 & 0.14 & 0.09 & 0.036 & 0.010 & 0.98 & 0.56 & 6.31 & 147 \\
\hline $2 \sigma$ & 0.55 & 0.21 & 0.39 & 0.05 & 34.92 & 3.75 & 1.13 & 0.57 & 0.51 & 0.280 & 0.265 & 1.47 & 0.32 & 1.37 & 160 \\
\hline Minimum & 0.02 & 0.01 & 0.11 & 0.02 & ND & ND & 0.11 & 0.01 & 0.04 & ND & ND & 0.13 & 0.19 & 4.73 & 6 \\
\hline Maximum & 1.38 & 0.55 & 1.34 & 0.17 & 167.50 & 12.92 & 2.97 & 1.65 & 2.41 & 0.643 & 1.248 & 3.75 & 1.08 & 8.57 & 269 \\
\hline \multicolumn{16}{|c|}{ Rolesville Granite $(n=52)$} \\
\hline Mean & 0.27 & 0.09 & 0.47 & 0.05 & 1.86 & 0.44 & 0.88 & 0.28 & 0.16 & 0.125 & 0.044 & 0.90 & 0.59 & 6.11 & 155 \\
\hline Median & 0.14 & 0.05 & 0.43 & 0.04 & 0.23 & 0.11 & 0.68 & 0.15 & 0.11 & 0.094 & 0.007 & 0.57 & 0.58 & 6.02 & 173 \\
\hline $2 \sigma$ & 0.64 & 0.20 & 0.35 & 0.03 & 0.90 & 1.69 & 1.25 & 0.74 & 0.23 & 0.232 & 0.141 & 1.68 & 0.32 & 1.28 & 159 \\
\hline Minimum & 0.03 & 0.01 & 0.11 & 0.02 & ND & ND & 0.23 & 0.01 & 0.04 & ND & ND & 0.13 & 0.22 & 4.73 & 13 \\
\hline Maximum & 1.38 & 0.55 & 1.25 & 0.12 & 58.32 & 5.12 & 2.97 & 1.65 & 0.61 & 0.460 & 0.332 & 3.75 & 1.08 & 7.47 & 269 \\
\hline \multicolumn{16}{|c|}{ Raleigh Gneiss $(n=24)$} \\
\hline Mean & 0.25 & 0.14 & 0.38 & 0.06 & 6.85 & 1.71 & 0.69 & 0.17 & 0.11 & 0.079 & 0.085 & 1.00 & 0.56 & 6.43 & 76 \\
\hline Median & 0.19 & 0.12 & 0.32 & 0.07 & 0.63 & 0.44 & 0.46 & 0.13 & 0.08 & 0.011 & 0.021 & 1.05 & 0.54 & 6.36 & 38 \\
\hline $2 \sigma$ & 0.40 & 0.19 & 0.42 & 0.05 & 1.41 & 5.90 & 1.12 & 0.24 & 0.20 & 0.321 & 0.511 & 1.02 & 0.41 & 1.3 & 144 \\
\hline Minimum & 0.08 & 0.02 & 0.21 & 0.03 & ND & 0.05 & 0.23 & 0.06 & 0.05 & ND & 0.003 & 0.31 & 0.19 & 5.54 & 6 \\
\hline Maximum & 0.98 & 0.38 & 1.07 & 0.12 & 51.71 & 12.92 & 2.74 & 0.51 & 0.53 & 0.643 & 1.248 & 2.31 & 1.07 & 8.24 & 188 \\
\hline \multicolumn{16}{|c|}{ Metasedimentary and metavolcanic rocks $(n=34)$} \\
\hline Mean & 0.36 & 0.18 & 0.50 & 0.06 & 6.99 & 1.01 & 0.78 & 0.18 & 0.22 & 0.075 & 0.038 & 1.41 & 0.55 & 6.63 & 118 \\
\hline Median & 0.28 & 0.17 & 0.45 & 0.06 & 0.23 & 0.12 & 0.68 & 0.12 & 0.08 & 0.004 & 0.012 & 1.40 & 0.53 & 6.51 & 131 \\
\hline $2 \sigma$ & 0.48 & 0.19 & 0.43 & 0.06 & 3.22 & 4.21 & 0.96 & 0.31 & 0.87 & 0.331 & 0.185 & 1.21 & 0.28 & 1.42 & 143 \\
\hline Minimum & 0.02 & 0.02 & 0.23 & 0.03 & ND & ND & 0.11 & 0.02 & 0.05 & ND & ND & 0.25 & 0.30 & 5.12 & 9 \\
\hline Maximum & 0.91 & 0.43 & 1.34 & 0.17 & 167.50 & 9.23 & 1.83 & 0.55 & 2.41 & 0.638 & 0.502 & 2.92 & 0.83 & 8.57 & 263 \\
\hline
\end{tabular}


Table 2

Major and trace element concentrations and $\mathrm{pH}$

\begin{tabular}{|c|c|c|c|c|c|c|c|c|c|c|c|c|c|c|c|c|c|c|c|c|}
\hline Group & $\begin{array}{l}\text { Well } \\
\text { ID }\end{array}$ & $\begin{array}{l}\text { Sampling } \\
\text { date }\end{array}$ & Rock type & $\begin{array}{l}\text { Well } \\
\text { depth } \\
(\mathrm{m})\end{array}$ & $\begin{array}{l}\text { Casing } \\
\text { depth } \\
(\mathrm{m})\end{array}$ & $\mathrm{pH}$ & $\begin{array}{l}\text { DO } \\
(\mu \mathrm{M})\end{array}$ & $\begin{array}{l}\mathrm{Mg} \\
(\mathrm{mM})\end{array}$ & $\begin{array}{l}\mathrm{Ca} \\
(\mathrm{mM})\end{array}$ & $\begin{array}{l}\mathrm{Sr} \\
(\mu \mathrm{M})\end{array}$ & $\begin{array}{l}\mathrm{Ba} \\
(\mu \mathrm{M})\end{array}$ & $\begin{array}{l}\mathrm{Na} \\
(\mathrm{mM})\end{array}$ & $\begin{array}{l}\mathrm{K} \\
(\mathrm{mM})\end{array}$ & $\begin{array}{l}\mathrm{Cl}^{-} \\
(\mathrm{mM})\end{array}$ & $\begin{array}{l}\mathrm{NO}_{3}^{-} \\
(\mathrm{mM})\end{array}$ & $\begin{array}{l}\mathrm{SO}_{4}^{2-} \\
(\mathrm{mM})\end{array}$ & $\begin{array}{l}\mathrm{HCO}_{3}^{-} \\
(\mathrm{mM})\end{array}$ & $\begin{array}{l}\mathrm{SiO}_{2} \\
(\mathrm{mM})\end{array}$ & $\begin{array}{l}\mathrm{Mn} \\
(\mu \mathrm{M})\end{array}$ & $\begin{array}{l}\mathrm{Fe} \\
(\mu \mathrm{M})\end{array}$ \\
\hline $1 \mathrm{a}$ & 20 & 19 Jan 2007 & $\begin{array}{l}\text { Metasedimentary/ } \\
\text { Metavolcanic }\end{array}$ & 124 & 18 & 8.57 & 127 & 0.27 & 0.24 & 0.58 & 0.32 & 0.36 & 0.09 & 0.06 & 0.008 & 0.006 & 1.37 & 0.56 & 0.11 & 0.41 \\
\hline $1 \mathrm{a}$ & 22 & 21 Jan 2007 & $\begin{array}{l}\text { Metasedimentary/ } \\
\text { Metavolcanic }\end{array}$ & 125 & 20 & 6.32 & 89 & 0.20 & 0.50 & 0.39 & 0.07 & 0.57 & 0.07 & 0.08 & 0.066 & 0.039 & 1.90 & 0.41 & 0.04 & 0.25 \\
\hline $1 \mathrm{a}$ & 23 & 21 Jan 2007 & $\begin{array}{l}\text { Metasedimentary/ } \\
\text { Metavolcanic }\end{array}$ & 179 & 18 & 7.50 & 87 & 0.43 & 0.91 & 1.56 & 0.35 & 0.53 & 0.17 & 0.42 & 0.109 & 0.028 & 2.92 & 0.45 & 0.05 & 0.18 \\
\hline $1 \mathrm{a}$ & 24 & 21 Jan 2007 & $\begin{array}{l}\text { Metasedimentary/ } \\
\text { Metavolcanic }\end{array}$ & 57 & 21 & 7.20 & 222 & 0.08 & 0.13 & 0.37 & 0.08 & 0.23 & 0.04 & 0.08 & 0.110 & 0.005 & 0.53 & 0.54 & 0.15 & 0.26 \\
\hline $1 \mathrm{a}$ & 25 & 21 Jan 2007 & $\begin{array}{l}\text { Metadiorite/ } \\
\text { Metagranodiorite }\end{array}$ & 107 & 14 & 6.79 & 145 & 0.32 & 0.80 & 1.55 & 0.93 & 0.70 & 0.09 & 0.38 & 0.028 & 0.126 & 2.28 & 0.70 & 0.32 & 0.34 \\
\hline $1 \mathrm{a}$ & 26 & 26 Jan 2007 & Ultramafic & 124 & 19 & 7.12 & 171 & 0.14 & 0.17 & 0.38 & 0.13 & 0.27 & 0.09 & 0.07 & 0.111 & 0.007 & 0.89 & 0.55 & 0.16 & 0.49 \\
\hline $1 \mathrm{a}$ & 27 & 26 Jan 2007 & $\begin{array}{l}\text { Metadiorite/ } \\
\text { Metagranodiorite }\end{array}$ & & & 6.54 & 240 & 0.11 & 0.16 & 0.50 & 0.05 & 0.29 & 0.03 & 0.10 & 0.162 & 0.004 & 1.16 & 0.37 & 0.15 & 0.23 \\
\hline $1 \mathrm{a}$ & 28 & 26 Jan 2007 & $\begin{array}{l}\text { Metasedimentary/ } \\
\text { Metavolcanic }\end{array}$ & 112 & 8 & 7.50 & 149 & 0.14 & 0.22 & 0.42 & 0.05 & 0.34 & 0.07 & 0.06 & 0.016 & 0.016 & 1.23 & 0.47 & 0.15 & 0.18 \\
\hline $1 \mathrm{a}$ & 29 & 26 Jan 2007 & $\begin{array}{l}\text { Metasedimentary/ } \\
\text { Metavolcanic }\end{array}$ & 92 & & 6.04 & 151 & 0.22 & 0.25 & 0.66 & 0.23 & 0.46 & 0.06 & 0.10 & 0.010 & 0.006 & 1.33 & 0.66 & 0.07 & 0.08 \\
\hline $1 \mathrm{a}$ & 30 & 26 Jan 2007 & $\begin{array}{l}\text { Metasedimentary/ } \\
\text { Metavolcanic }\end{array}$ & 81 & 15 & 6.20 & 180 & 0.23 & 0.72 & 1.05 & 0.12 & 0.47 & 0.06 & 0.39 & 0.495 & 0.004 & 2.37 & 0.51 & 0.12 & 0.21 \\
\hline $1 \mathrm{a}$ & 31 & 28 Jan 2007 & $\begin{array}{l}\text { Metadiorite/ } \\
\text { Metagranodiorite }\end{array}$ & 76 & 13 & 6.33 & 47 & 0.21 & 0.64 & 1.20 & 0.48 & 0.46 & 0.09 & 0.11 & 0.022 & 0.039 & 1.74 & 0.59 & 1.04 & 0.68 \\
\hline $1 \mathrm{a}$ & 32 & 28 Jan 2007 & $\begin{array}{l}\text { Metasedimentary/ } \\
\text { Metavolcanic }\end{array}$ & & & 6.21 & 134 & 0.33 & 0.56 & 1.87 & 0.56 & 0.67 & 0.09 & 1.01 & 0.602 & 0.007 & 1.02 & 0.50 & 0.03 & 0.31 \\
\hline $1 \mathrm{a}$ & 34 & 28 Jan 2007 & $\begin{array}{l}\text { Metasedimentary/ } \\
\text { Metavolcanic }\end{array}$ & 75 & 31 & 6.51 & 160 & 0.13 & 0.19 & 0.63 & 0.24 & 0.42 & 0.06 & 0.07 & 0.005 & 0.005 & 1.02 & 0.72 & 0.20 & ND \\
\hline $1 \mathrm{a}$ & 35 & 28 Jan 2007 & $\begin{array}{l}\text { Metadiorite/ } \\
\text { Metagranodiorite }\end{array}$ & 50 & 18 & 6.15 & 96 & 0.21 & 0.33 & 1.10 & 0.62 & 0.62 & 0.05 & 0.18 & 0.028 & 0.052 & 1.95 & 0.79 & 0.07 & 0.72 \\
\hline $1 \mathrm{a}$ & 36 & 02 Feb 2007 & $\begin{array}{l}\text { Metasedimentary/ } \\
\text { Metavolcanic }\end{array}$ & & & 6.62 & 263 & 0.11 & 0.12 & 0.50 & 0.03 & 0.31 & 0.04 & 0.08 & 0.007 & 0.004 & 0.76 & 0.43 & ND & ND \\
\hline $1 \mathrm{a}$ & 37 & 02 Feb 2007 & $\begin{array}{l}\text { Metasedimentary/ } \\
\text { Metavolcanic }\end{array}$ & & & 6.88 & 133 & 0.14 & 0.63 & 0.79 & 0.05 & 0.58 & 0.04 & 0.10 & 0.066 & 0.243 & 1.57 & 0.37 & 0.04 & 0.15 \\
\hline $1 \mathrm{a}$ & 38 & 02 Feb 2007 & $\begin{array}{l}\text { Metasedimentary/ } \\
\text { Metavolcanic }\end{array}$ & 81 & 19 & 7.45 & 32 & 0.20 & 0.91 & 1.41 & 0.13 & 0.93 & 0.07 & 0.09 & 0.002 & 0.501 & 2.07 & 0.42 & 0.18 & 0.46 \\
\hline $1 \mathrm{a}$ & 39 & 02 Feb 2007 & $\begin{array}{l}\text { Metasedimentary/ } \\
\text { Metavolcanic }\end{array}$ & 55 & 20 & 6.21 & 46 & 0.30 & 0.78 & 1.20 & 0.35 & 0.42 & 0.08 & 0.52 & 0.001 & 0.095 & 2.08 & 0.55 & 9.24 & 16.95 \\
\hline $1 \mathrm{a}$ & 40 & 02 Feb 2007 & $\begin{array}{l}\text { Metasedimentary/ } \\
\text { Metavolcanic }\end{array}$ & 69 & 12 & 6.13 & 218 & 0.26 & 0.50 & 1.64 & 0.54 & 0.67 & 0.07 & 0.44 & 0.638 & 0.015 & 1.25 & 0.63 & 0.06 & 0.22 \\
\hline $1 \mathrm{a}$ & 48 & 11 Feb 2007 & $\begin{array}{l}\text { Metasedimentary/ } \\
\text { Metavolcanic }\end{array}$ & 93 & 11 & 5.78 & & 0.10 & 0.22 & 0.58 & 0.06 & 0.32 & 0.03 & 0.09 & 0.025 & 0.005 & 1.42 & 0.41 & 0.07 & 0.35 \\
\hline $1 \mathrm{a}$ & 49 & 11 Feb 2007 & $\begin{array}{l}\text { Metasedimentary/ } \\
\text { Metavolcanic }\end{array}$ & 185 & 15 & 6.31 & & 0.31 & 0.35 & 1.20 & 0.18 & 0.48 & 0.06 & 0.14 & 0.150 & 0.006 & 1.68 & 0.56 & 0.03 & 0.14 \\
\hline $1 b$ & 19 & 19 Jan 2007 & $\begin{array}{l}\text { Metasedimentary/ } \\
\text { Metavolcanic }\end{array}$ & 104 & 12 & 6.87 & 19 & 0.12 & 0.34 & 1.23 & 0.04 & 0.86 & 0.05 & 0.06 & ND & 0.010 & 1.75 & 0.29 & 0.17 & 0.16 \\
\hline $1 b$ & 41 & 09 Feb 2007 & $\begin{array}{l}\text { Metadiorite/ } \\
\text { Metagranodiorite }\end{array}$ & & & 7.16 & 68 & 0.17 & 0.30 & 0.74 & 0.11 & 0.40 & 0.06 & 0.06 & 0.001 & 0.049 & 1.35 & 0.56 & 0.78 & 1.36 \\
\hline $1 b$ & 42 & 09 Feb 2007 & Raleigh gneiss & 46 & 29 & 7.08 & 13 & 0.34 & 0.56 & 0.94 & 0.06 & 0.54 & 0.06 & 0.09 & 0.001 & 0.039 & 2.31 & 0.53 & 1.16 & 8.37 \\
\hline $1 b$ & 43 & 09 Feb 2007 & $\begin{array}{l}\text { Metasedimentary/ } \\
\text { Metavolcanic }\end{array}$ & 104 & & 6.51 & & 0.15 & 0.28 & 0.50 & 0.22 & 0.44 & 0.04 & 0.07 & 0.001 & 0.009 & 1.30 & 0.81 & 0.02 & 0.03 \\
\hline $1 b$ & 44 & 09 Feb 2007 & $\begin{array}{l}\text { Metasedimentary/ } \\
\text { Metavolcanic }\end{array}$ & 70 & 38 & 6.32 & 12 & 0.20 & 0.29 & 0.33 & 0.10 & 0.39 & 0.06 & 0.06 & ND & 0.030 & 1.93 & 0.62 & 3.91 & 15.45 \\
\hline $1 b$ & 45 & 09 Feb 2007 & $\begin{array}{l}\text { Metasedimentary/ } \\
\text { Metavolcanic }\end{array}$ & 92 & 28 & 5.99 & 184 & 0.02 & 0.06 & 0.16 & 0.16 & 0.35 & 0.04 & 0.05 & 0.001 & 0.005 & 0.58 & 0.65 & 0.00 & 0.32 \\
\hline $1 b$ & 50 & 16 Feb 2007 & $\begin{array}{l}\text { Metasedimentary/ } \\
\text { Metavolcanic }\end{array}$ & & & 5.60 & 127 & 0.30 & 0.49 & 1.76 & 0.44 & 1.34 & 0.07 & 2.41 & 0.080 & 0.025 & 1.42 & 0.79 & 0.08 & 0.03 \\
\hline $1 b$ & 51 & 16 Feb 2007 & $\begin{array}{l}\text { Metasedimentary/ } \\
\text { Metavolcanic }\end{array}$ & 99 & 47 & 8.28 & 10 & 0.17 & 0.32 & 1.02 & 0.02 & 0.71 & 0.06 & 0.07 & 0.001 & 0.016 & 1.67 & 0.32 & 0.06 & ND \\
\hline $1 b$ & 52 & 16 Feb 2007 & Raleigh gneiss & 113 & 37 & 7.82 & 38 & 0.13 & 0.40 & 1.79 & 0.09 & 1.06 & 0.07 & 0.07 & 0.001 & 0.032 & 1.87 & 0.48 & 0.55 & 0.59 \\
\hline $1 b$ & 53 & 16 Feb 2007 & $\begin{array}{l}\text { Metasedimentary/ } \\
\text { Metavolcanic }\end{array}$ & 75 & 32 & 6.29 & 27 & 0.12 & 0.10 & 0.35 & 0.04 & 0.44 & 0.03 & 0.06 & 0.001 & 0.022 & 1.10 & 0.69 & 3.08 & 10.99 \\
\hline $1 b$ & 54 & 16 Feb 2007 & $\begin{array}{l}\text { Metadiorite/ } \\
\text { Metagranodiorite }\end{array}$ & & & 6.30 & 168 & 0.05 & 0.08 & 0.28 & 0.09 & 0.21 & 0.02 & 0.06 & 0.008 & 0.003 & 0.48 & 0.64 & 0.06 & 0.00 \\
\hline $1 b$ & 55 & 23 Feb 2007 & $\begin{array}{l}\text { Metasedimentary/ } \\
\text { Metavolcanic }\end{array}$ & & & 6.70 & 130 & 0.17 & 0.34 & 0.65 & 0.11 & 0.42 & 0.09 & 0.06 & 0.002 & 0.005 & 1.45 & 0.56 & 0.19 & 0.25 \\
\hline $1 b$ & 56 & 23 Feb 2007 & $\begin{array}{l}\text { Metasedimentary/ } \\
\text { Metavolcanic }\end{array}$ & 86 & 20 & 6.20 & 164 & 0.04 & 0.09 & 0.29 & 0.16 & 0.31 & 0.03 & 0.06 & 0.001 & 0.005 & 0.64 & 0.60 & 0.23 & ND \\
\hline $1 b$ & 57 & 23 Feb 2007 & $\begin{array}{l}\text { Metasedimentary/ } \\
\text { Metavolcanic }\end{array}$ & 75 & 24 & 7.21 & 36 & 0.18 & 0.66 & 0.69 & 0.27 & 0.46 & 0.04 & 0.07 & 0.001 & 0.051 & 2.19 & 0.81 & 3.66 & 2.00 \\
\hline $1 b$ & 58 & 23 Feb 2007 & Raleigh gneiss & 39 & & 6.29 & 15 & 0.23 & 0.39 & 0.69 & 0.17 & 0.32 & 0.07 & 0.12 & 0.001 & 0.079 & 1.58 & 0.52 & 5.33 & 22.97 \\
\hline $1 b$ & 59 & 23 Feb 2007 & $\begin{array}{l}\text { Metasedimentary/ } \\
\text { Metavolcanic }\end{array}$ & & & 5.12 & 208 & 0.02 & 0.03 & 0.17 & 0.41 & 0.48 & 0.05 & 0.18 & 0.124 & ND & 0.24 & 0.47 & 0.11 & 0.20 \\
\hline $1 b$ & 60 & 25 Feb 2007 & $\begin{array}{l}\text { Metasedimentary/ } \\
\text { Metavolcanic }\end{array}$ & 80 & 25 & 6.65 & 166 & 0.15 & 0.23 & 0.48 & 0.03 & 0.47 & 0.03 & 0.05 & ND & 0.011 & 1.09 & 0.83 & 0.65 & 0.11 \\
\hline
\end{tabular}


Table 2 (continued)

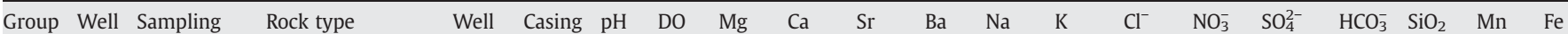
ID date depth depth (m) (m)

\begin{tabular}{|c|c|c|c|c|c|c|c|c|c|c|c|c|c|c|c|c|c|c|c|c|}
\hline $1 \mathrm{~b}$ & 61 & 25 Feb 2007 & $\begin{array}{l}\text { Metasedimentary/ } \\
\text { Metavolcanic }\end{array}$ & & & 6.95 & 83 & 0.24 & 0.51 & 1.30 & 0.03 & 0.62 & 0.09 & 0.10 & ND & 0.012 & 2.06 & 0.47 & 0.08 & 0.40 \\
\hline $1 b$ & 62 & 25 Feb 2007 & $\begin{array}{l}\text { Metasedimentary/ } \\
\text { Metavolcanic }\end{array}$ & & & 7.26 & 81 & 0.24 & 0.43 & 0.78 & 0.19 & 0.42 & 0.07 & 0.09 & ND & 0.016 & 1.90 & 0.43 & 1.37 & 1.10 \\
\hline $1 b$ & 63 & 25 Feb 2007 & $\begin{array}{l}\text { Metasedimentary/ } \\
\text { Metavolcanic }\end{array}$ & 76 & 32 & 6.10 & 188 & 0.06 & 0.04 & 0.18 & 0.03 & 0.27 & 0.03 & 0.07 & 0.028 & 0.017 & 0.26 & 0.52 & 0.22 & 0.31 \\
\hline $1 b$ & 74 & 04 Mar 2007 & $\begin{array}{l}\text { Metasedimentary/ } \\
\text { Metavolcanic }\end{array}$ & 69 & 33 & 6.91 & 153 & 0.23 & 0.25 & 0.56 & 0.04 & 0.50 & 0.07 & 0.06 & ND & 0.012 & 1.44 & 0.48 & 0.05 & 0.21 \\
\hline $1 b$ & 75 & 04 Mar 2007 & $\begin{array}{l}\text { Metasedimentary/ } \\
\text { Metavolcanic }\end{array}$ & 70 & 57 & 6.81 & 33 & 0.23 & 0.22 & 0.46 & 0.02 & 0.45 & 0.06 & 0.06 & ND & 0.032 & 1.38 & 0.70 & 3.18 & 18.37 \\
\hline 2 & 11 & 14 Jan 2007 & Raleigh gneiss & 24 & 20 & 6.45 & 11 & 0.32 & 0.18 & 0.41 & 0.25 & 0.22 & 0.10 & 0.08 & 0.002 & 0.046 & 1.23 & 1.07 & 12.93 & 51.71 \\
\hline 2 & 64 & 02 Mar 2007 & Raleigh gneiss & 73 & 10 & 6.86 & 30 & 0.17 & 9.26 & 28.37 & 0.13 & 7.34 & 0.07 & 0.92 & ND & 13.261 & 0.31 & 0.24 & 1.42 & 1.62 \\
\hline 2 & 65 & 02 Mar 2007 & $\begin{array}{l}\text { Metasedimentary/ } \\
\text { Metavolcanic }\end{array}$ & 75 & 44 & 6.32 & 25 & 0.09 & 0.18 & 0.95 & 0.35 & 0.28 & 0.04 & 0.06 & ND & 0.024 & 0.92 & 0.47 & 6.68 & 167.52 \\
\hline 2 & 66 & 02 Mar 2007 & Raleigh gneiss & 61 & 16 & 7.01 & 14 & 0.15 & 0.40 & 0.94 & 0.12 & 0.40 & 0.07 & 0.08 & ND & 0.106 & 1.37 & 0.60 & 4.48 & 7.88 \\
\hline 2 & 67 & 02 Mar 2007 & Raleigh gneiss & 52 & 15 & 6.49 & 43 & 0.14 & 0.32 & 0.78 & 0.08 & 0.37 & 0.07 & 0.07 & 0.009 & 0.075 & 1.14 & 0.68 & 0.23 & 0.09 \\
\hline 2 & 68 & 09 Mar 2007 & Raleigh gneiss & & 28 & 6.39 & 172 & 0.11 & 0.20 & 0.79 & 0.13 & 0.30 & 0.04 & 0.08 & 0.033 & 0.003 & 0.88 & 0.57 & 0.06 & 0.69 \\
\hline 2 & 69 & 09 Mar 2007 & Raleigh gneiss & & & 6.30 & 13 & 0.14 & 0.13 & 0.38 & 0.13 & 0.33 & 0.07 & 0.06 & 0.001 & 0.076 & 1.06 & 0.90 & 0.86 & 1.74 \\
\hline 2 & 70 & 09 Mar 2007 & Raleigh gneiss & 122 & 31 & 5.94 & 172 & 0.08 & 0.12 & 0.36 & 0.07 & 0.31 & 0.06 & 0.08 & 0.086 & 0.006 & 1.44 & 0.64 & 0.05 & ND \\
\hline 2 & 71 & 09 Mar 2007 & Raleigh gneiss & 37 & 20 & 7.05 & 7 & 0.14 & 0.19 & 0.46 & 0.30 & 0.33 & 0.07 & 0.05 & ND & 0.038 & 1.05 & 0.65 & 3.20 & 20.97 \\
\hline 2 & 72 & 04 Mar 2007 & Raleigh gneiss & & & 6.04 & 154 & 0.04 & 0.11 & 0.30 & 0.14 & 0.21 & 0.03 & 0.09 & 0.077 & 0.038 & 0.45 & 0.30 & 0.43 & ND \\
\hline 2 & 73 & 04 Mar 2007 & Raleigh gneiss & 69 & 28 & 5.54 & 186 & 0.04 & 0.10 & 0.36 & 0.11 & 0.29 & 0.04 & 0.07 & 0.030 & 0.003 & 0.53 & 0.52 & 0.32 & 0.63 \\
\hline 2 & 76 & 04 Mar 2007 & Raleigh gneiss & 64 & 29 & 6.36 & 9 & 0.19 & 0.25 & 0.59 & 0.46 & 0.28 & 0.05 & 0.06 & ND & 0.017 & 1.18 & 0.62 & 4.93 & 25.99 \\
\hline 2 & 77 & 18 Mar 2007 & Raleigh gneiss & & & 6.44 & 22 & 0.14 & 0.17 & 0.46 & 0.16 & 0.23 & 0.09 & 0.06 & ND & 0.096 & 0.81 & 0.89 & 1.55 & 12.17 \\
\hline 2 & 78 & 18 Mar 2007 & Raleigh gneiss & 93 & 21 & 5.97 & 175 & 0.04 & 0.08 & 0.27 & 0.10 & 0.22 & 0.04 & 0.07 & 0.067 & 0.004 & 0.42 & 0.48 & 0.09 & 0.15 \\
\hline 2 & 79 & 18 Mar 2007 & Raleigh gneiss & 75 & 31 & 6.39 & 119 & 0.12 & 0.21 & 0.77 & 0.18 & 0.29 & 0.07 & 0.07 & 0.011 & 0.006 & 1.06 & 0.63 & 0.28 & 0.37 \\
\hline 2 & 80 & 18 Mar 2007 & Raleigh gneiss & & & 5.94 & 173 & 0.07 & 0.23 & 0.96 & 0.20 & 0.41 & 0.07 & 0.20 & 0.643 & 0.006 & 0.31 & 0.49 & 0.15 & 1.04 \\
\hline 2 & 81 & 18 Mar 2007 & Raleigh gneiss & 165 & 14 & 6.11 & 95 & 0.10 & 0.16 & 0.48 & 0.06 & 0.37 & 0.05 & 0.12 & 0.085 & 0.019 & 0.76 & 0.55 & 0.22 & 0.16 \\
\hline 2 & 82 & 25 Mar 2007 & Raleigh gneiss & 61 & 43 & 6.16 & & 0.03 & 0.08 & 0.39 & 0.07 & 0.32 & 0.03 & 0.07 & 0.014 & 0.003 & 0.65 & 0.60 & 0.06 & ND \\
\hline 2 & 83 & 25 Mar 2007 & Raleigh gneiss & & & 5.56 & & 0.06 & 0.13 & 0.34 & 0.28 & 0.28 & 0.03 & 0.15 & 0.368 & 0.003 & 0.37 & 0.23 & 0.16 & 0.22 \\
\hline 2 & 84 & 25 Mar 2007 & Raleigh gneiss & 70 & 24 & 6.46 & & 0.11 & 0.15 & 0.49 & 0.16 & 0.24 & 0.05 & 0.07 & 0.022 & 0.003 & 0.82 & 0.47 & 0.05 & 0.28 \\
\hline 2 & 85 & 25 Mar 2007 & Raleigh gneiss & 73 & 42 & 8.24 & 13 & 0.38 & 0.97 & 2.78 & 0.11 & 0.91 & 0.12 & 0.16 & 0.005 & 1.248 & 1.29 & 0.33 & 1.79 & 1.45 \\
\hline 2 & 86 & 25 Mar 2007 & Raleigh gneiss & & & 5.87 & & 0.08 & 0.23 & 0.41 & 0.51 & 0.45 & 0.12 & 0.53 & 0.364 & 0.021 & 0.42 & 0.19 & 0.47 & 0.09 \\
\hline $3 a$ & 7 & 12 Jan 2007 & Granite & 55 & 20 & 6.31 & 176 & 0.02 & 0.07 & 0.43 & 0.20 & 0.35 & 0.05 & 0.05 & 0.001 & 0.003 & 0.64 & 0.83 & 0.03 & 0.17 \\
\hline $3 a$ & 8 & 14 Jan 2007 & Granite & 46 & 7 & 6.80 & 188 & 0.12 & 0.33 & 1.03 & 0.07 & 0.53 & 0.04 & 0.10 & 0.198 & 0.046 & 1.03 & 0.60 & 0.11 & ND \\
\hline $3 a$ & 9 & 14 Jan 2007 & Granite & 18 & 13 & 5.74 & 213 & 0.05 & 0.18 & 0.73 & 0.13 & 0.41 & 0.05 & 0.11 & 0.234 & 0.001 & 0.44 & 0.53 & 0.15 & 0.07 \\
\hline $3 a$ & 10 & 14 Jan 2007 & Granite & 38 & 16 & 6.01 & 215 & 0.06 & 0.18 & 0.93 & 0.14 & 0.33 & 0.06 & 0.08 & 0.061 & 0.003 & 0.74 & 0.53 & 2.45 & 3.61 \\
\hline $3 a$ & 12 & 14 Jan 2007 & Granite & & & 6.33 & 178 & 0.20 & 0.24 & 0.63 & 0.30 & 0.43 & 0.03 & 0.16 & 0.198 & 0.016 & 0.91 & 0.75 & 0.07 & 0.07 \\
\hline $3 a$ & 13 & 15 Jan 2007 & Granite & 49 & 29 & 6.50 & 13 & 0.05 & 0.08 & 0.49 & 0.31 & 0.45 & 0.06 & 0.04 & ND & 0.005 & 1.42 & 1.08 & 1.17 & 58.32 \\
\hline $3 a$ & 14 & 15 Jan 2007 & Granite & 61 & 18 & 5.77 & 171 & 0.03 & 0.10 & 0.79 & 0.30 & 0.42 & 0.04 & 0.17 & 0.107 & 0.004 & 0.52 & 0.72 & 0.14 & 0.34 \\
\hline $3 a$ & 16 & 15 Jan 2007 & Granite & 92 & 18 & 5.68 & 268 & 0.01 & 0.03 & 0.31 & 0.11 & 0.19 & 0.04 & 0.05 & 0.011 & 0.002 & 0.29 & 0.52 & 0.00 & 0.34 \\
\hline $3 a$ & 17 & 15 Jan 2007 & Granite & 75 & 20 & 6.02 & 260 & 0.02 & 0.08 & 0.44 & 0.20 & 0.25 & 0.05 & 0.08 & 0.117 & 0.004 & 0.41 & 0.54 & 0.05 & 0.80 \\
\hline $3 a$ & 18 & 19 Jan 2007 & Granite & 147 & 21 & 6.87 & 118 & 0.04 & 0.20 & 0.95 & 0.25 & 0.53 & 0.05 & 0.08 & 0.062 & 0.085 & 0.78 & 0.46 & 0.35 & ND \\
\hline $3 a$ & 21 & 19 Jan 2007 & Granite & 43 & 24 & 6.00 & 136 & 0.02 & 0.07 & 0.43 & 0.20 & 0.33 & 0.05 & 0.05 & 0.004 & 0.003 & 0.61 & 0.68 & 0.23 & 1.85 \\
\hline $3 a$ & 33 & 28 Jan 2007 & Granite & 55 & 28 & 6.62 & 248 & 0.02 & 0.06 & 0.35 & 0.12 & 0.33 & 0.04 & 0.05 & 0.004 & 0.004 & 0.53 & 0.67 & 0.10 & 0.27 \\
\hline $3 a$ & 47 & 11 Feb 2007 & Granite & 52 & & 6.61 & 15 & 0.22 & 0.73 & 2.31 & 1.09 & 0.59 & 0.04 & 0.15 & 0.002 & 0.150 & 2.17 & 0.70 & 1.68 & 6.81 \\
\hline $3 a$ & 89 & 09 May 2007 & Granite & & & 5.28 & 200 & 0.03 & 0.08 & 0.52 & 0.31 & 0.43 & 0.06 & 0.36 & 0.192 & 0.002 & 0.21 & 0.32 & 0.09 & 0.10 \\
\hline $3 a$ & 90 & 09 May 2007 & Granite & 69 & 25 & 6.04 & 33 & 0.06 & 0.13 & 0.43 & 0.12 & 0.30 & 0.05 & 0.10 & 0.074 & 0.141 & 0.60 & 0.61 & 0.84 & 1.14 \\
\hline $3 a$ & 92 & 11 May 2007 & Granite & & & 6.48 & 259 & 0.04 & 0.09 & 0.41 & 0.02 & 0.36 & 0.04 & 0.06 & 0.011 & 0.006 & 0.58 & 0.79 & 0.08 & ND \\
\hline $3 a$ & 93 & 11 May 2007 & Granite & 58 & 31 & 6.17 & 214 & 0.02 & 0.07 & 0.42 & 0.17 & 0.27 & 0.06 & 0.07 & 0.039 & 0.004 & 0.46 & 0.68 & 0.11 & 0.10 \\
\hline $3 a$ & 95 & 20 May 2007 & Granite & & & 5.25 & 228 & 0.02 & 0.03 & 0.20 & 0.28 & 0.11 & 0.05 & 0.07 & 0.049 & ND & 0.25 & 0.22 & 0.06 & ND \\
\hline $3 a$ & 96 & 20 May 2007 & Granite & & & 5.81 & 135 & 0.06 & 0.15 & 0.99 & 0.15 & 0.57 & 0.04 & 0.14 & 0.237 & 0.002 & 0.65 & 0.81 & 0.10 & 0.10 \\
\hline $3 a$ & 97 & 20 May 2007 & Granite & 45 & 24 & 5.93 & 255 & 0.02 & 0.06 & 0.28 & 0.18 & 0.29 & 0.05 & 0.12 & 0.184 & 0.003 & 0.30 & 0.50 & 0.02 & 0.03 \\
\hline $3 a$ & 99 & 21 Oct 2007 & Granite & 92 & & 5.87 & 236 & 0.04 & 0.10 & 0.43 & 0.06 & 0.39 & 0.04 & 0.05 & 0.010 & 0.005 & 0.56 & 0.80 & 0.06 & 0.13 \\
\hline $3 b$ & 1 & 14 Dec 2006 & Granite & 343 & 12 & 6.30 & 17 & 0.07 & 0.24 & 0.85 & 0.13 & 0.69 & 0.03 & 0.28 & 0.156 & 0.021 & 2.09 & 0.47 & 0.52 & 1.83 \\
\hline $3 b$ & 2 & 14 Dec 2006 & Granite & 119 & 19 & 6.92 & 44 & 0.09 & 0.30 & 0.91 & 0.36 & 0.45 & 0.03 & 0.10 & 0.086 & 0.011 & 1.18 & 0.55 & 0.26 & 0.07 \\
\hline $3 b$ & 3 & 14 Dec 2006 & Granite & 275 & 18 & 5.96 & 68 & 0.11 & 0.51 & 0.81 & 0.06 & 0.55 & 0.04 & 0.28 & 0.304 & 0.086 & 1.10 & 0.43 & 0.30 & 0.07 \\
\hline $3 b$ & 4 & 14 Dec 2006 & Granite & & & 7.44 & 26 & 0.34 & 0.84 & 3.01 & 1.64 & 1.25 & 0.04 & 0.10 & 0.002 & 0.087 & 3.24 & 0.54 & 1.19 & 1.58 \\
\hline $3 b$ & 5 & 12 Jan 2007 & Granite & 127 & 10 & 5.23 & 241 & 0.02 & 0.08 & 0.41 & 0.12 & 0.40 & 0.03 & 0.10 & 0.187 & 0.003 & 0.35 & 0.57 & 0.06 & 0.34 \\
\hline $3 b$ & 6 & 12 Jan 2007 & Granite & 104 & 9 & 7.10 & 12 & 0.55 & 1.38 & 2.47 & 1.55 & 0.60 & 0.05 & 0.14 & 0.007 & 0.189 & 3.76 & 0.45 & 5.12 & 2.76 \\
\hline $3 b$ & 15 & 15 Jan 2007 & Granite & 55 & 13 & 6.43 & 139 & 0.08 & 0.26 & 0.92 & 0.04 & 0.55 & 0.02 & 0.09 & 0.118 & 0.076 & 1.16 & 0.70 & 0.12 & 0.25 \\
\hline $3 b$ & 46 & 11 Feb 2007 & Granite & & & 5.02 & 35 & 0.05 & 0.14 & 0.87 & 0.73 & 0.62 & 0.12 & 0.44 & 0.346 & 0.029 & 0.28 & 0.47 & 0.08 & 0.62 \\
\hline $3 b$ & 87 & 09 May 2007 & Granite & & & 5.37 & 147 & 0.07 & 0.19 & 1.44 & 0.35 & 0.78 & 0.07 & 0.61 & 0.318 & 0.041 & 0.38 & 0.58 & 0.09 & ND \\
\hline $3 b$ & 88 & 09 May 2007 & Granite & 21 & 6 & 5.46 & 235 & 0.02 & 0.07 & 0.38 & 0.03 & 0.47 & 0.03 & 0.10 & 0.346 & 0.006 & 0.15 & 0.49 & 0.14 & 0.26 \\
\hline $3 b$ & 91 & 11 May 2007 & Granite & & & 6.10 & 177 & 0.03 & 0.26 & 0.53 & 0.14 & 0.42 & 0.03 & 0.10 & 0.051 & 0.048 & 0.79 & 0.38 & 0.41 & 3.85 \\
\hline $3 b$ & 94 & 13 May 2007 & Granite & & & 5.46 & 103 & 0.02 & 0.18 & 0.55 & 1.38 & 0.34 & 0.08 & 0.24 & 0.206 & 0.008 & 0.41 & 0.31 & 0.41 & ND \\
\hline $3 b$ & 98 & 10 Sep 2007 & Granite & & & 6.59 & 158 & 0.19 & 0.52 & 1.07 & 0.40 & 0.51 & 0.03 & 0.15 & 0.062 & 0.095 & 1.51 & 0.57 & 0.05 & 0.18 \\
\hline $3 c$ & 100 & 21 Oct 2007 & Granite & 31 & & 5.68 & 253 & 0.08 & 0.13 & 0.59 & 0.03 & 0.57 & 0.04 & 0.17 & 0.354 & 0.001 & 0.29 & 0.67 & 0.05 & 0.18 \\
\hline $3 c$ & 101 & 21 Oct 2007 & Granite & 47 & & 5.75 & 133 & 0.06 & 0.14 & 0.56 & 0.20 & 0.30 & 0.04 & 0.08 & 0.142 & 0.108 & 0.24 & 0.42 & 1.13 & 3.95 \\
\hline $3 c$ & 102 & 21 Oct 2007 & Granite & 124 & 16 & 6.75 & 69 & 0.19 & 1.35 & 2.15 & 0.14 & 0.57 & 0.04 & 0.18 & 0.051 & 0.130 & 3.19 & 0.51 & 0.11 & 0.12 \\
\hline $3 c$ & 103 & 26 Oct 2007 & Granite & & & & 206 & 0.09 & 0.13 & 0.50 & 0.13 & 0.33 & 0.04 & 0.11 & 0.104 & 0.009 & 0.48 & 0.58 & 0.05 & 0.23 \\
\hline $3 c$ & 104 & 26 Oct 2007 & Granite & 69 & 17 & 7.10 & 162 & 0.11 & 0.98 & 1.63 & 0.15 & 0.53 & 0.04 & 0.17 & 0.069 & 0.087 & 2.03 & 0.54 & 1.37 & 0.26 \\
\hline $3 c$ & 105 & 26 Oct 2007 & Granite & 73 & 19 & 7.25 & 36 & 0.24 & 0.96 & 2.27 & 0.01 & 0.67 & 0.04 & 0.21 & 0.030 & 0.332 & 2.16 & 0.50 & 0.04 & 0.08 \\
\hline $3 c$ & 106 & 11 Nov 2007 & Granite & 58 & 16 & 5.76 & 175 & 0.05 & 0.17 & 1.00 & 0.29 & 0.57 & 0.06 & 0.16 & 0.460 & 0.001 & 0.38 & 0.73 & 0.12 & 0.05 \\
\hline
\end{tabular}


Table 2 (continued)

\begin{tabular}{|c|c|c|c|c|c|c|c|c|c|c|c|c|c|c|c|c|c|c|c|c|}
\hline Group & $\begin{array}{l}\text { Well } \\
\text { ID }\end{array}$ & $\begin{array}{l}\text { Sampling } \\
\text { date }\end{array}$ & Rock type & $\begin{array}{l}\text { Well } \\
\text { depth } \\
\text { (m) }\end{array}$ & $\begin{array}{l}\text { Casing } \\
\text { depth } \\
\text { (m) }\end{array}$ & $\mathrm{pH}$ & $\begin{array}{l}\text { DO } \\
(\mu \mathrm{M})\end{array}$ & $\begin{array}{l}\mathrm{Mg} \\
(\mathrm{mM})\end{array}$ & $\begin{array}{l}\mathrm{Ca} \\
(\mathrm{mM})\end{array}$ & $\begin{array}{l}\mathrm{Sr} \\
(\mu \mathrm{M})\end{array}$ & $\begin{array}{l}\mathrm{Ba} \\
(\mu \mathrm{M})\end{array}$ & $\begin{array}{l}\mathrm{Na} \\
(\mathrm{mM})\end{array}$ & $\begin{array}{l}\mathrm{K} \\
(\mathrm{mM})\end{array}$ & $\begin{array}{l}\mathrm{Cl}^{-} \\
(\mathrm{mM})\end{array}$ & $\begin{array}{l}\mathrm{NO}_{3}^{-} \\
(\mathrm{mM})\end{array}$ & $\begin{array}{l}\mathrm{SO}_{4}^{2-} \\
(\mathrm{mM})\end{array}$ & $\begin{array}{l}\mathrm{HCO}_{3}^{-} \\
(\mathrm{mM})\end{array}$ & $\begin{array}{l}\mathrm{SiO}_{2} \\
(\mathrm{mM})\end{array}$ & $\begin{array}{l}\mathrm{Mn} \\
(\mu \mathrm{M})\end{array}$ & $\begin{array}{l}\mathrm{Fe} \\
(\mu \mathrm{M})\end{array}$ \\
\hline $3 c$ & 107 & 11 Nov 2007 & Granite & 44 & 18 & 7.47 & 14 & 0.38 & 0.70 & 0.99 & 0.11 & 0.53 & 0.04 & 0.14 & 0.014 & 0.117 & 2.35 & 0.64 & 1.82 & 0.24 \\
\hline $3 c$ & 108 & 11 Nov 2007 & Granite & 130 & & 6.03 & 175 & 0.12 & 0.50 & 2.11 & 0.17 & 0.67 & 0.05 & 0.36 & 0.112 & 0.262 & 0.94 & 0.52 & 0.11 & 0.05 \\
\hline $3 c$ & 109 & 11 Nov 2007 & Granite & 40 & 19 & 6.04 & 236 & 0.03 & 0.08 & 0.41 & 0.09 & 0.40 & 0.04 & 0.13 & 0.153 & 0.005 & 0.29 & 0.73 & 0.09 & 1.15 \\
\hline $3 c$ & 110 & 11 Nov 2007 & Granite & 61 & 14 & 4.73 & 163 & 0.05 & 0.09 & 0.84 & 0.86 & 0.53 & 0.07 & 0.42 & 0.196 & 0.004 & 0.13 & 0.31 & 0.28 & 0.21 \\
\hline $3 c$ & 111 & 18 Nov 2007 & Granite & 45 & 16 & 5.10 & 200 & 0.04 & 0.09 & 0.44 & 0.10 & 0.38 & 0.03 & 0.12 & 0.043 & 0.010 & 0.43 & 0.65 & ND & 0.06 \\
\hline $3 c$ & 112 & 18 Nov 2007 & Granite & 87 & 34 & 5.68 & 172 & 0.10 & 0.20 & 1.29 & 0.24 & 0.61 & 0.06 & 0.31 & 0.340 & 0.005 & 0.58 & 0.75 & 0.09 & 0.15 \\
\hline $3 c$ & 113 & 18 Nov 2007 & Granite & & & 5.90 & 156 & 0.07 & 0.14 & 0.75 & 0.14 & 0.42 & 0.05 & 0.10 & 0.021 & ND & 0.76 & 0.66 & 0.35 & 0.62 \\
\hline $3 c$ & 114 & 18 Nov 2007 & Granite & & & 5.91 & 213 & 0.06 & 0.13 & 0.63 & 0.13 & 0.43 & 0.04 & 0.17 & 0.232 & ND & 0.38 & 0.68 & 0.09 & 0.59 \\
\hline $3 c$ & 115 & 18 Nov 2007 & Granite & 86 & & 6.54 & 213 & 0.05 & 0.13 & 0.35 & 0.05 & 0.36 & 0.02 & 0.10 & 0.047 & 0.009 & 0.57 & 0.81 & 0.10 & 0.33 \\
\hline $3 c$ & 116 & 07 Dec 2007 & Granite & & & & 88 & 0.05 & 0.14 & 0.65 & 0.09 & 0.44 & 0.04 & 0.09 & 0.063 & 0.010 & 0.68 & 0.78 & 0.40 & 0.33 \\
\hline $3 c$ & 117 & 07 Dec 2007 & Granite & 83 & & & 216 & 0.03 & 0.09 & 0.55 & 0.06 & 0.35 & 0.02 & 0.17 & 0.102 & 0.001 & 0.33 & 0.50 & 0.04 & 1.80 \\
\hline
\end{tabular}

ND indicates not detected; these were treated as zero concentrations in data analysis.

sampling materials. However, one blank each evaluating bottles and filtration indicate minimal effects on major cations, Fe, Mn, and U. Major anion $\left(\mathrm{Cl}^{-}, \mathrm{NO}_{3}^{-}, \mathrm{SO}_{4}^{2-}\right)$ concentrations were determined by ion chromatography, and bicarbonate concentrations were determined by titration to $\mathrm{pH} 4.5$.

\subsection{Data handling}

Speciation and saturation index values were calculated using the PHREEQC geochemical code (Parkhurst and Appelo, 1999; Post, 2006) modified with Ra speciation coefficients from Langmuir and Riese (1985). Bedrock geology at each sampling site was assigned from 1:100,000-scale map data based on Clark et al. (2004). Correlation coefficients reported in the text are Spearman rank coefficients.

\section{Results}

\subsection{Major ions, trace elements, and redox-sensitive elements}

The overall chemical composition of the investigated groundwater from the different lithological settings is dominated by $\mathrm{Na}-\mathrm{Ca}-\mathrm{Mg}$
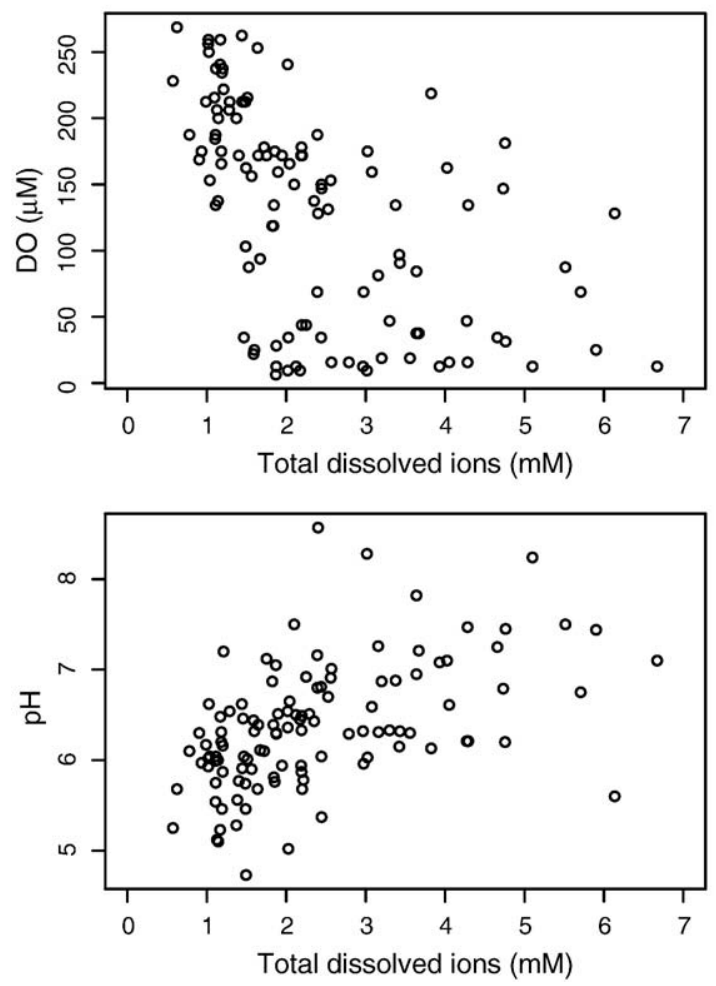

Fig. 2. Relationships between $\mathrm{pH}$, dissolved oxygen concentration, and total ions. bicarbonate, except for a few samples with anions dominated by a combination of bicarbonate and nitrate, chloride, or sulfate (Table 1 ). One anomalous high-sulfate water, well 64 in Group 2 (Table 2), was excluded from plots and correlations due to its non-representative chemistry. Average major element chemistry exhibits few apparent differences between the three main rock types in the study area. Wells in the Raleigh Gneiss exhibit lower average DO concentrations than the other rock types. Also, the Rolesville Granite wells exhibit lower average $\mathrm{Ca}, \mathrm{Mg}$, $\mathrm{K}$, and bicarbonate concentrations than the other rock
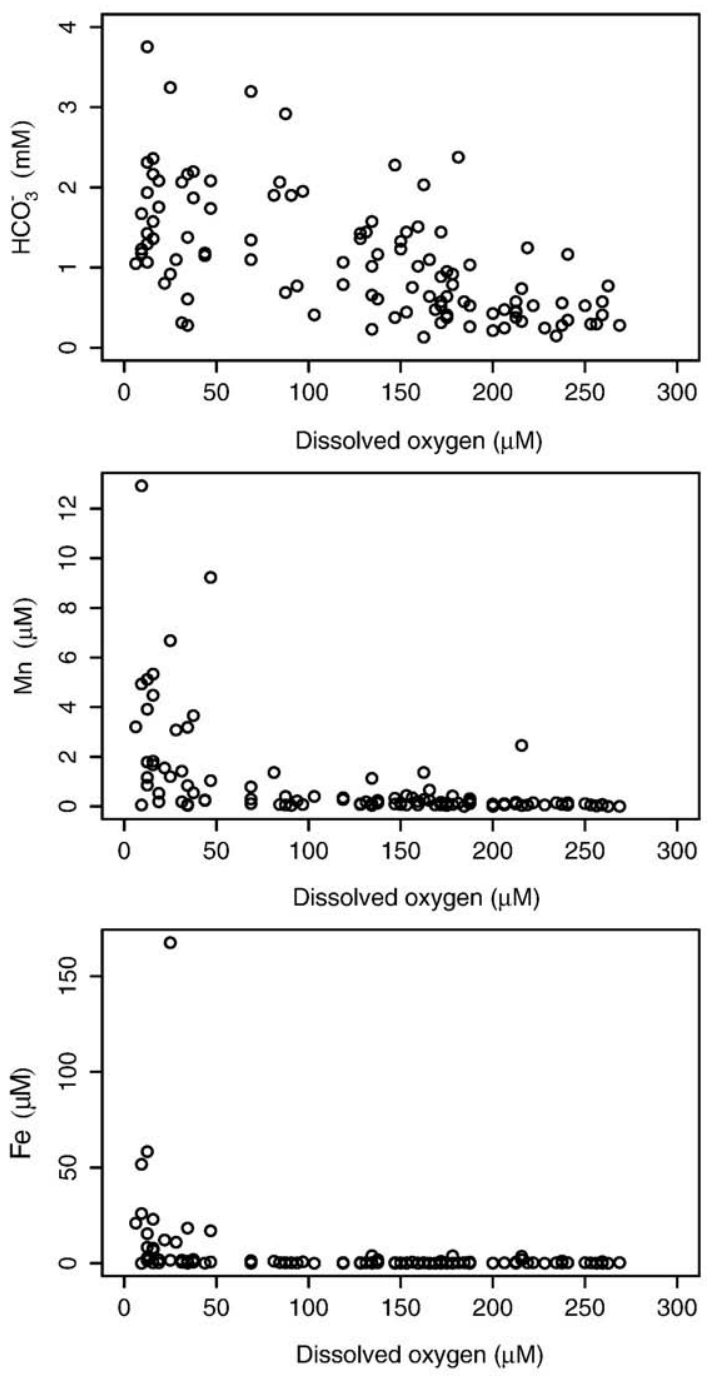

Fig. 3. Relationship between dissolved oxygen and other redox-sensitive solutes. 
types (Table 1). However, concentrations of major elements and redox-sensitive elements vary significantly between wells. Most samples fall along a continuum between (1) slightly acidic ( $\mathrm{pH}$ 5.06.0 ), oxic, low-total dissolved solids (TDS) waters, and (2) nearneutral, oxic to anoxic, higher-TDS waters (Fig. 2). These trends illustrate the linked nature of the major element and redox chemistry of the waters. Dissolved oxygen concentration is inversely associated with $\mathrm{Mn}, \mathrm{Fe}$, and $\mathrm{HCO}_{3}^{-}$concentrations (Fig. 3), reflecting the sensitivity of these solutes to redox processes. As barite $\left(\mathrm{BaSO}_{4}\right)$ supersaturation is a potential sink for Ra, saturation index values were calculated from major element chemistry and Ba concentrations; however, waters in this study rarely approach barite saturation (median-1.9; range -3.4 to 0.2 ), indicating a negligible role for barite.

\subsection{Radionuclide activities in water}

In contrast to major element chemistry, radionuclide activities exhibit large variation between the Rolesville Granite and the other rock types in the study area. Median radon-222 activity (249 Bq L ${ }^{-1}$ ) in the Rolesville Granite exceeds the other rock units by about one order of magnitude (Table 3), and median radium-226 activity (18.6 $\left.\mathrm{mBq} \mathrm{L}{ }^{-1}\right)$ is about a factor of 2 higher in the Rolesville Granite (Table 3). Median uranium concentration (5 nM) is 1-2 orders of magnitude higher in the Rolesville Granite than in the other rock types (Table 3). Also, ${ }^{222} \mathrm{Rn}$ activity shows an inverse relationship with total dissolved ions (TDI) and direct relationship with DO, whereas ${ }^{226} \mathrm{Ra}$ is directly associated with TDI and inversely with DO (Fig. 4). Relative to EPA drinking water standards applied to public water systems, $77 \%$ of samples from the Rolesville Granite exceed the proposed alternative maximum contaminant level (MCL) for ${ }^{222} \mathrm{Rn}$ of $148 \mathrm{~Bq} \mathrm{~L}^{-1} .10 \%$ exceed the MCL for uranium of $126 \mathrm{nM}\left(30 \mu \mathrm{g} \mathrm{L}^{-1}\right)$, and $6 \%$ exceed the MCL for
${ }^{226} \mathrm{Ra}+{ }^{228} \mathrm{Ra}$ of $185 \mathrm{mBq} \mathrm{L}{ }^{-1}$. Radionuclide levels above the MCLs are uncommon in the other rock types (Table 4). In the Rolesville Granite, $\mathrm{U}$ is correlated with $\mathrm{pH}(\rho=0.52), \mathrm{HCO}_{3}^{-}(\rho=0.49)$, Ca $(\rho=0.48)$, and sulfate $(\rho=0.47)$. However, no clear relationships were observed between $U$ and redox conditions or other radionuclides except for a relatively weak association with ${ }^{226} \mathrm{Ra}(\rho=0.36)$. Because radionuclide activities and activity ratios are often lognormally distributed (e.g. King et al., 1982), geometric mean and geometric standard deviation are presented in Table 3.

\subsection{Radionuclide content of granite rock samples}

Gamma spectrometric analysis of crushed Rolesville Granite bulk samples indicates ${ }^{226} \mathrm{Ra}$ activity from 20.8 to $53.5 \mathrm{~Bq} \mathrm{~kg}^{-1}$ and ${ }^{228} \mathrm{Ra}$ from 42.4 to $72.1 \mathrm{~Bq} \mathrm{~kg}{ }^{-1}$. This produces ${ }^{228} \mathrm{Ra} /{ }^{226} \mathrm{Ra}$ of solids ranging from 0.99 to 2.68 . These activities and activity ratios vary within each core and between the two cores analyzed (Table 5 ).

\section{Discussion}

\subsection{Major element and redox chemistry of potential Ra sinks}

The high silica concentrations, dominance of bicarbonate among the dissolved anions, and correlation between pH and TDI ( $\rho=0.55$; Fig. 2 ) indicate that most of the solutes in the investigated groundwater are derived from silicate weathering, which consumes protons, contributes cations, and converts soil gas $\mathrm{CO}_{2}$ to bicarbonate (Drever, 1997). Bicarbonate may also be derived from organic carbon oxidation or calcite dissolution, although the latter should be insignificant in longexposed granites in areas where denudation rates are low, such as the southeastern United States (White et al., 1999). The mass balance of

Table 3

Summary of radionuclide activities and activity ratios

\begin{tabular}{|c|c|c|c|c|c|c|c|c|c|}
\hline All data $(n=117)$ & ${ }^{222} \mathrm{Rn}\left(\mathrm{Bq} \mathrm{L}{ }^{-1}\right)$ & ${ }^{224} \mathrm{Ra}\left(\mathrm{mBq} \mathrm{L}{ }^{-1}\right)$ & ${ }^{226} \mathrm{Ra}\left(\mathrm{mBq} \mathrm{L}{ }^{-1}\right)$ & ${ }^{228} \mathrm{Ra}\left(\mathrm{mBq} \mathrm{L}{ }^{-1}\right)$ & $\mathrm{U}(\mathrm{nM})$ & ${ }^{222} \mathrm{Rn} /{ }^{224} \mathrm{Ra}$ & ${ }^{222} \mathrm{Rn} /{ }^{226} \mathrm{Ra}$ & ${ }^{228} \mathrm{Ra} /{ }^{226} \mathrm{Ra}$ & ${ }^{224} \mathrm{Ra} /{ }^{228} \mathrm{Ra}$ \\
\hline Mean & 170 & 17.4 & 21.4 & 17.9 & 25 & 22,420 & 17,710 & 1.17 & 1.03 \\
\hline Median & 77 & 8.5 & 9.4 & 9.3 & 1 & 11,190 & 9,023 & 0.95 & 0.96 \\
\hline $2 \sigma$ & 451 & 73.0 & 81.5 & 66.7 & 172 & 63,214 & 56,879 & 1.59 & 0.93 \\
\hline Geometric mean $^{a}$ & 76 & 8.6 & 9.8 & 9.5 & 3 & 8,693 & 7,603 & 0.94 & 0.93 \\
\hline 2 geom std dev $^{a}$ & 2.9 & 2.1 & 2.4 & 2.0 & 4.4 & 3.1 & 2.9 & 1.37 & 0.9 \\
\hline Minimum & $<3$ & 0.6 & 0.7 & ND & ND & 194 & 201 & 0.15 & 0.20 \\
\hline Maximum & 1,113 & 324.0 & 348.6 & 234.1 & 593 & 170,900 & 247,800 & 4.26 & 2.60 \\
\hline \multicolumn{10}{|c|}{ Rolesville granite $(n=52)$} \\
\hline Mean & 335 & 26.4 & 36.9 & 26.4 & 55 & 36,450 & 24,700 & 0.76 & 1.08 \\
\hline Median & 249 & 11.6 & 18.6 & 12.3 & 5 & 17,910 & 12,060 & 0.68 & 0.98 \\
\hline $2 \sigma$ & 503 & 100.6 & 109.1 & 89.3 & 245 & 80,981 & 76,245 & 1.24 & 0.89 \\
\hline Geometric mean & 249 & 14.0 & 22.4 & 13.9 & 9 & 17,776 & 11,114 & 0.62 & 1.01 \\
\hline 2 geom std dev & 1.7 & 1.9 & 1.9 & 2.0 & 4.3 & 2.8 & 2.8 & 1.24 & 0.8 \\
\hline Minimum & 23 & 2.4 & 1.4 & 2.0 & ND & 331 & 307 & 0.15 & 0.43 \\
\hline Maximum & 1,113 & 324.0 & 348.6 & 234.1 & 593 & 170,900 & 247,800 & 4.26 & 2.47 \\
\hline \multicolumn{10}{|c|}{ Raleigh gneiss $(n=24)$} \\
\hline Mean & 44 & 11.5 & 11.1 & 12.3 & $<1$ & 13,680 & 10,880 & 1.23 & 0.89 \\
\hline Median & 30 & 5.6 & 6.0 & 5.5 & ND & 6,904 & 6,142 & 0.98 & 0.91 \\
\hline $2 \sigma$ & 79 & 44.0 & 42.0 & 42.3 & 2 & 34,947 & 28,712 & 1.44 & 0.71 \\
\hline Geometric mean & 31 & 5.4 & 6.1 & 6.7 & 2 & 5,673 & 5,071 & 1.07 & 0.82 \\
\hline 2 geom std dev & 2.0 & 2.3 & 1.9 & 2.1 & 2.4 & 3.1 & 2.8 & 1.05 & 0.8 \\
\hline Minimum & $<3$ & 0.7 & 0.9 & ND & ND & 194 & 201 & 0.51 & 0.35 \\
\hline Maximum & 152 & 108.7 & 104.9 & 102.7 & 5 & 76,890 & 60,170 & 3.11 & 1.86 \\
\hline \multicolumn{10}{|c|}{ Metasedimentary and metavolcanic rocks $(n=34)$} \\
\hline Mean & 35 & 9.8 & 8.3 & 10.8 & 2 & 6,839 & 12,130 & 1.68 & 1.12 \\
\hline Median & 31 & 6.7 & 4.0 & 6.6 & 1 & 4,530 & 9,693 & 1.69 & 0.99 \\
\hline $2 \sigma$ & 58 & 25.7 & 28.9 & 34.1 & 9 & 13,183 & 25,629 & 1.56 & 1.09 \\
\hline Geometric mean & 25 & 6.6 & 4.6 & 7.0 & 1 & 3,672 & 5,594 & 1.49 & 0.99 \\
\hline 2 geom std dev & 2.1 & 1.7 & 1.9 & 1.8 & 3.2 & 2.6 & 3.0 & 1.04 & 1.1 \\
\hline Minimum & $<3$ & 0.6 & 0.7 & ND & ND & 245 & 247 & 0.39 & 0.20 \\
\hline Maximum & 96 & 76.0 & 83.3 & 96.3 & 17 & 23,470 & 50,830 & 3.53 & 2.60 \\
\hline
\end{tabular}

ND indicates not detected.

a Samples below detection excluded from geometric mean and geometric standard deviation. 

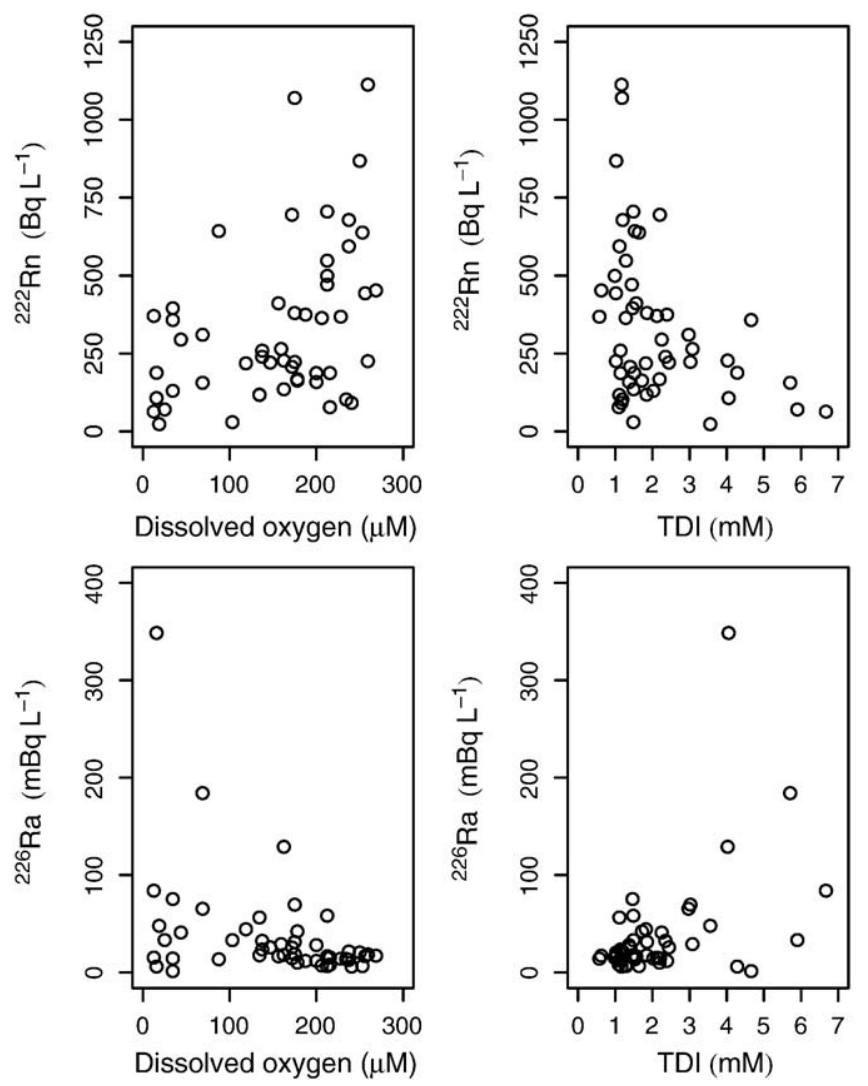

Fig. 4. Relationship between dissolved oxygen, total ions, and activities of ${ }^{222} \mathrm{Rn}$ and ${ }^{226} \mathrm{Ra}$ in Rolesville Granite groundwater.

dissolved oxygen (up to $\sim 0.3 \mathrm{mM}$ at atmospheric saturation, ranging to near zero in some samples) suggests that a large proportion of bicarbonate (median $0.98 \mathrm{mM}$ for all samples, $0.57 \mathrm{mM}$ in the Rolesville Granite) is derived from the open system of unsaturated zone $\mathrm{CO}_{2}$ rather than organic carbon oxidation in the saturated zone. Consequently, the majority of cations are also derived from the shallow weathering zone. The drinking water wells sampled in this study commonly contain openhole intervals tens of meters long and produce water from an unknown configuration of fractures. However, once groundwater passes through the weathered zone (soil and saprolite) into the fracture network of relatively unweathered bedrock, where the open-hole interval is located (Daniel and Dahlen, 2002), the effect of mineral weathering on major element chemistry is expected to be minimal due to the relatively short residence time in the fractures and the high ratio of water to mineral surfaces. Therefore, major solute composition is determined during the early stages of groundwater evolution.

Major species that are sensitive to redox conditions in groundwater include DO, nitrate, Mn, Fe, sulfate, and bicarbonate. The large variations in DO, Fe, and Mn concentrations (Fig. 3) indicate that redox processes play an important role in influencing water chemistry. In fractured crystalline rocks, variations in redox condition may be due to abiotic reductants including iron(II) in biotite (Gascoyne, 1997) or iron (II) and sulfide in pyrite (Gascoyne, 1997; Tarits et al., 2006). Biotite is abundant in the Rolesville Granite (Kosecki and Fodor, 1997), and pyrite has been observed in a pluton of similar age and setting in South Carolina (Speer et al., 1981). Redox processes may also be driven by organic carbon oxidation (Banwart et al., 1996, 1999; Gascoyne, 2004). In the saturated zone, organic carbon could be oxidized to bicarbonate by aerobic respiration or other terminal electron-accepting processes including denitrification, Mn oxide reduction, and Fe oxide reduction.

Mn concentrations over $2 \mu \mathrm{M}$ and Fe concentrations over $10 \mu \mathrm{M}$ are almost invariably associated with DO concentrations below $50 \mu \mathrm{M}$ (Fig. 3). The elevated Mn and Fe in anoxic fractures indicate conditions favorable for reductive dissolution of Mn and Fe oxides. No evidence for sulfate reduction, such as a sulfide odor, was observed, suggesting that $\mathrm{Mn}$ and/or Fe oxide reduction represent the most reducing biogeochemical conditions that prevail in a significant number of wells. Finally, elevated bicarbonate concentrations, when associated with decreasing DO concentrations (Fig. 2), may indicate the effects of organic carbon oxidation, although alternative mechanisms of producing bicarbonate and consuming DO, discussed above, may be of equal or greater significance.

Our results indicate negative correlation of DO with total dissolved ion (TDI) concentrations ( $\rho=-0.63$; Fig. 2 ), which suggests that DO is affected by some combination of organic carbon oxidation and oxidation of minerals such as biotite or pyrite related to weathering. Although determination of the rates of these processes is beyond the scope of this paper, other studies indicate a negative relationship between DO and apparent residence time in Piedmont groundwater and overall residence times of about 10-50 a (e.g. Nelms and Harlow, 2003; D. Harned, USGS, Raleigh, NC, unpub. data, 2007). These relationships suggest the potential importance of relatively slow processes such as mineral oxidation on water chemistry, as the role of abiotic reductants in controlling groundwater redox conditions is expected to be more important in older, slowly-circulating groundwater systems (Gascoyne, 1997, 2004). In comparison, consumption of dissolved oxygen by biogeochemical reactions in bedrock fractures can be as rapid as several days (Puigdomenech et al., 2000).

\subsection{Radium and radon isotopes}

Overall activities of radium are higher in the Rolesville Granite than in the metasedimentary and metavolcanic rocks and in the Raleigh Gneiss (Table 3). Also, Ra isotope activities co-occur, that is, high ${ }^{226} \mathrm{Ra}$ from the ${ }^{238} \mathrm{U}$ decay series tends to be accompanied by high ${ }^{224} \mathrm{Ra}$ and ${ }^{228} \mathrm{Ra}$ from the ${ }^{232} \mathrm{Th}$ series $\left(\rho=0.78,0.79\right.$, and 0.87 for the ${ }^{226} \mathrm{Ra}-{ }^{228} \mathrm{Ra}$, ${ }^{224} \mathrm{Ra}-{ }^{226} \mathrm{Ra}$, and ${ }^{224} \mathrm{Ra}-{ }^{228} \mathrm{Ra}$ pairs, respectively, in the overall data set). In groundwater from the Rolesville Granite, where Ra activities are higher and relative counting error lower, the strongest correlations $(\rho \geq 0.40)$ of ${ }^{226} \mathrm{Ra}$ with other elements are with $\mathrm{Mn}>\mathrm{Ca}>\mathrm{Ba}>\mathrm{DO}$ ( $\rho=0.51,0.41,0.41$, and -0.40 , respectively). The strongest correlations of ${ }^{228} \mathrm{Ra}$ are with $\mathrm{Ba}>\mathrm{K}>\mathrm{Mn}(\rho=0.57,0.49$, and 0.40 , respectively). Overall, Ra is positively correlated with $\mathrm{Ba}$ and to a lesser degree with other alkaline earth metals. Ra is also correlated with redox-sensitive solutes such as DO and $\mathrm{Mn}$ in a consistent fashion: more reduced conditions are generally associated with higher Ra (Fig. 4). Although acidity ( $\mathrm{pH}$ below 5) may significantly inhibit Ra adsorption (Nirdosh et al., 1990; Moon et al., 2003), and pH variations are associated with variations in DO and TDI (Fig. 2), pH does not exhibit strong correlations with Ra or Rn $\left(\rho=0.07,0.23\right.$, and 0.00 between $\mathrm{pH}$ and ${ }^{222} \mathrm{Rn},{ }^{226} \mathrm{Ra}$, and ${ }^{228} \mathrm{Ra}$, respectively, in the Rolesville Granite). Mn oxides and clays exhibit negative surface charge above pH 5 (Stumm and Morgan, 1996), so within the $\mathrm{pH}$ range in this study, Ra adsorption is relatively unaffected by variations in $\mathrm{pH}$. Also, because anion concentrations in these waters are 1-2 orders of magnitude below levels expected to significantly complex Ra (Langmuir and Riese, 1985), aqueous complexation of Ra is negligible. Speciation calculations indicate that virtually all aqueous Ra is present as $\mathrm{Ra}^{2+}$ (in Rolesville Granite, median 99.7\% as $\mathrm{Ra}^{2+}$, range 91.5\%-100\%). The remaining $\mathrm{Ra}$ is complexed as $\mathrm{RaSO}_{4}^{0}$. Results are essentially identical for $\mathrm{Ba}$ (median $98.1 \%$ as $\mathrm{Ba}^{2+}$, range $79.2-100 \%$ ). Thus, Ra occurs primarily as $\mathrm{Ra}^{2+}$, which is strongly subject to adsorption at near-neutral $\mathrm{pH}$.

\subsection{1. ${ }^{228} \mathrm{Ra} /{ }^{226} \mathrm{Ra}$ activity ratio}

The ${ }^{228} \mathrm{Ra} /{ }^{226} \mathrm{Ra}$ ratio in groundwater may be expected to resemble the ${ }^{232} \mathrm{Th} /{ }^{238} \mathrm{U}$ ratio of the host rock (Asikainen, 1981a; Porcelli, 2008) assuming secular equilibrium within the rock. Overall variations in ${ }^{228} \mathrm{Ra} /{ }^{226} \mathrm{Ra}$ occur with rock type; water samples from the Rolesville Granite exhibit lower median ${ }^{228} \mathrm{Ra} /{ }^{226} \mathrm{Ra}$ than the Raleigh Gneiss and 
Table 4

Radionuclide activities and activity ratios

\begin{tabular}{|c|c|c|c|c|c|c|c|c|c|c|c|c|c|}
\hline Group & $\begin{array}{l}\text { Well } \\
\text { ID }\end{array}$ & $\begin{array}{l}\mathrm{U} \\
(\mathrm{nM})\end{array}$ & ${ }^{224} \mathrm{Ra}\left(\mathrm{mBq} \mathrm{L} \mathrm{L}^{-1}\right)$ & ${ }^{226} \mathrm{Ra}\left(\mathrm{mBq} \mathrm{L} \mathrm{L}^{-1}\right)$ & ${ }^{228} \mathrm{Ra}\left(\mathrm{mBq} \mathrm{L}^{-1}\right)$ & ${ }^{222} \mathrm{Rn}^{\mathrm{a}}\left(\mathrm{Bq} \mathrm{L} \mathrm{L}^{-1}\right)$ & Group & $\begin{array}{l}\text { Well } \\
\text { ID }\end{array}$ & $\begin{array}{l}\mathrm{U} \\
(\mathrm{nM})\end{array}$ & ${ }^{224} \mathrm{Ra}\left(\mathrm{mBq} \mathrm{L} \mathrm{L}^{-1}\right)$ & ${ }^{226} \mathrm{Ra}\left(\mathrm{mBq} \mathrm{L}^{-1}\right)$ & ${ }^{228} \mathrm{Ra}\left(\mathrm{mBq} \mathrm{L} \mathrm{L}^{-1}\right)$ & ${ }^{222} \mathrm{Rn}\left(\mathrm{Bq} \mathrm{L} \mathrm{L}^{-1}\right)$ \\
\hline $1 \mathrm{a}$ & 20 & 1 & $11.3 \pm 1.1$ & $5.1 \pm 0.9$ & $9.4 \pm 1.9$ & 49 & 2 & 81 & 3 & $6.8 \pm 0.4$ & $7.2 \pm 1.1$ & $5.3 \pm 2.1$ & 152 \\
\hline $1 \mathrm{a}$ & 22 & 17 & $6.1 \pm 0.7$ & $5.5 \pm 0.7$ & $5.6 \pm 2.4$ & 3 & 2 & 82 & ND & $2.4 \pm 0.3$ & $2.9 \pm 0.9$ & $1.8 \pm 3.0$ & 78 \\
\hline $1 \mathrm{a}$ & 23 & 5 & $7.0 \pm 0.8$ & $4.0 \pm 0.8$ & $6.1 \pm 2.3$ & 23 & 2 & 83 & ND & $1.8 \pm 0.3$ & $3.5 \pm 0.7$ & $3.0 \pm 2.6$ & 30 \\
\hline $1 \mathrm{a}$ & 24 & ND & $3.4 \pm 0.4$ & $4.9 \pm 0.9$ & $2.9 \pm 1.9$ & 79 & 2 & 84 & ND & $0.8 \pm 0.2$ & $2.2 \pm 0.8$ & $2.2 \pm 2.4$ & 13 \\
\hline $1 \mathrm{a}$ & 25 & 1 & $12.5 \pm 0.9$ & $6.5 \pm 1.1$ & $10.7 \pm 3.3$ & $<3$ & 2 & 85 & ND & $4.1 \pm 0.5$ & $5.0 \pm 0.9$ & $3.7 \pm 9.2$ & $20^{\mathrm{b}}$ \\
\hline $1 \mathrm{a}$ & 26 & 1 & $1.2 \pm 0.2$ & $1.5 \pm 0.9$ & $4.2 \pm 2.9$ & 52 & 2 & 86 & ND & $108.7 \pm 6.6$ & $104.9 \pm 3.3$ & $102.8 \pm 8.9$ & 21 \\
\hline $1 \mathrm{a}$ & 27 & ND & $2.3 \pm 0.3$ & $2.6 \pm 0.9$ & $4.9 \pm 1.8$ & 40 & $3 a$ & 7 & 6 & $9.3 \pm 1.0$ & $19.0 \pm 1.3$ & $10.3 \pm 2.1$ & 1070 \\
\hline $1 \mathrm{a}$ & 28 & 1 & $3.3 \pm 0.4$ & $1.6 \pm 0.6$ & $3.2 \pm 2.3$ & 7 & $3 a$ & 8 & 102 & $11.1 \pm 0.6$ & $11.9 \pm 1.1$ & $10.0 \pm 1.5$ & 375 \\
\hline $1 \mathrm{a}$ & 29 & 1 & $6.4 \pm 0.7$ & $4.8 \pm 1.0$ & $17.0 \pm 5.9$ & 47 & $3 a$ & 9 & 80 & $21.1 \pm 2.4$ & $58.4 \pm 3.2$ & $20.8 \pm 1.7$ & 705 \\
\hline $1 a$ & 30 & 14 & $2.5 \pm 0.3$ & $0.7 \pm 0.9$ & $1.3 \pm 1.9$ & 32 & $3 a$ & 10 & 19 & $9.5 \pm 0.7$ & $15.6 \pm 1.4$ & $10.9 \pm 1.5$ & 187 \\
\hline $1 a$ & 31 & 6 & $13.3 \pm 0.9$ & $7.0 \pm 0.9$ & $13.8 \pm 2.9$ & 27 & $3 a$ & 12 & 2 & $12.2 \pm 0.6$ & $10.1 \pm 1.0$ & $9.3 \pm 2.2$ & 168 \\
\hline $1 \mathrm{a}$ & 32 & ND & $12.1 \pm 0.7$ & $6.2 \pm 1.1$ & $12.7 \pm 2.3$ & $<3$ & $3 a$ & 13 & ND & $4.9 \pm 0.6$ & $15.3 \pm 1.5$ & $7.9 \pm 1.9$ & 371 \\
\hline $1 \mathrm{a}$ & 34 & ND & $1.8 \pm 0.3$ & $3.7 \pm 0.6$ & ND & 40 & $3 a$ & 14 & 2 & $18.9 \pm 1.0$ & $25.8 \pm 1.8$ & $19.8 \pm 3.9$ & 207 \\
\hline $1 \mathrm{a}$ & 35 & 1 & $1.6 \pm 0.2$ & $2.2 \pm 0.7$ & $4.6 \pm 2.7$ & 19 & $3 a$ & 16 & ND & $14.4 \pm 1.2$ & $17.3 \pm 1.7$ & $12.4 \pm 2.5$ & 453 \\
\hline $1 \mathrm{a}$ & 36 & ND & $5.7 \pm 0.6$ & $3.7 \pm 0.7$ & $9.3 \pm 3.8$ & 95 & $3 a$ & 17 & 5 & $22.9 \pm 1.4$ & $18.6 \pm 1.4$ & $9.4 \pm 2.1$ & 225 \\
\hline $1 \mathrm{a}$ & 37 & 7 & $7.1 \pm 0.7$ & $1.6 \pm 0.8$ & $3.1 \pm 3.3$ & 50 & $3 a$ & 18 & 83 & $45.8 \pm 1.5$ & $44.5 \pm 2.5$ & $31.2 \pm 5.9$ & 219 \\
\hline $1 \mathrm{a}$ & 38 & 16 & $20.7 \pm 1.3$ & $20.5 \pm 1.6$ & $8.0 \pm 6.2$ & 5 & $3 a$ & 21 & 6 & $11.7 \pm 1.1$ & $23.6 \pm 1.8$ & $12.1 \pm 2.3$ & 259 \\
\hline $1 a$ & 39 & 1 & $13.2 \pm 1.2$ & $22.1 \pm 1.4$ & $14.3 \pm 4.3$ & 14 & $3 a$ & 33 & 8 & $7.5 \pm 0.9$ & $20.6 \pm 1.6$ & $17.0 \pm 5.9$ & 868 \\
\hline $1 \mathrm{a}$ & 40 & ND & $2.4 \pm 0.3$ & $1.2 \pm 0.9$ & $1.5 \pm 2.4$ & 11 & $3 a$ & 47 & 1 & $324.0 \pm 15.2$ & $348.6 \pm 7.6$ & $234.1 \pm 34.5$ & 107 \\
\hline $1 \mathrm{a}$ & 48 & 1 & $4.4 \pm 0.5$ & $2.6 \pm 0.6$ & $2.7 \pm 1.9$ & 37 & $3 a$ & 89 & 22 & $29.5 \pm 2.0$ & $28.2 \pm 1.7$ & $13.8 \pm 4.6$ & 159 \\
\hline $1 \mathrm{a}$ & 49 & 1 & $7.0 \pm 0.7$ & $5.0 \pm 0.8$ & $7.2 \pm 4.8$ & 81 & $3 a$ & 90 & 8 & $57.6 \pm 3.1$ & $75.4 \pm 2.9$ & $64.1 \pm 10.7$ & 395 \\
\hline $1 b$ & 19 & 2 & $13.7 \pm 0.8$ & $7.1 \pm 1.1$ & $12.3 \pm 4.2$ & 13 & $3 a$ & 92 & 28 & $6.5 \pm 1.1$ & $18.5 \pm 1.4$ & $5.0 \pm 3.2$ & 1113 \\
\hline $1 b$ & 41 & ND & $6.2 \pm 0.7$ & $7.3 \pm 1.1$ & $8.1 \pm 3.4$ & 12 & $3 a$ & 93 & ND & $9.2 \pm 0.8$ & $14.9 \pm 1.2$ & $14.3 \pm 4.3$ & 499 \\
\hline $1 b$ & 42 & ND & $13.0 \pm 0.9$ & $7.0 \pm 1.0$ & $21.8 \pm 5.0$ & 14 & $3 a$ & 95 & ND & $6.8 \pm 0.7$ & $14.2 \pm 1.4$ & $10.3 \pm 3.6$ & 368 \\
\hline $1 b$ & 43 & ND & $3.7 \pm 0.4$ & $2.6 \pm 1.0$ & $6.1 \pm 3.2$ & 3 & $3 a$ & 96 & ND & $8.6 \pm 0.8$ & $17.7 \pm 1.6$ & $11.1 \pm 3.7$ & 118 \\
\hline $1 b$ & 44 & ND & $10.2 \pm 1.0$ & $13.9 \pm 1.1$ & $14.8 \pm 4.9$ & $<3$ & $3 a$ & 97 & 2 & $16.9 \pm 1.6$ & $16.8 \pm 1.5$ & $17.6 \pm 5.4$ & 443 \\
\hline $1 b$ & 45 & ND & $4.7 \pm 0.6$ & $2.9 \pm 0.6$ & $3.7 \pm 2.4$ & 48 & $3 a$ & 99 & 36 & $9.8 \pm 1.7$ & $21.6 \pm 1.9$ & $10.1 \pm 5.7$ & 679 \\
\hline $1 b$ & 50 & ND & $8.5 \pm 1.0$ & $4.0 \pm 0.6$ & $5.2 \pm 1.9$ & 8 & $3 b$ & 1 & 1 & $9.2 \pm 0.9$ & $48.0 \pm 2.2$ & $14.3 \pm 6.0$ & 23 \\
\hline $1 b$ & 51 & 3 & $8.0 \pm 1.0$ & $5.0 \pm 0.9$ & $8.5 \pm 3.6$ & 58 & $3 b$ & 2 & 60 & $22.0 \pm 1.5$ & $41.1 \pm 2.7$ & $23.8 \pm 2.2$ & 295 \\
\hline $1 b$ & 52 & 5 & $22.1 \pm 1.7$ & $11.3 \pm 1.3$ & $31.2 \pm 7.1$ & 32 & $3 b$ & 3 & 340 & $22.0 \pm 2.6$ & $65.5 \pm 2.7$ & $15.1 \pm 3.2$ & 310 \\
\hline $1 b$ & 53 & 1 & $2.4 \pm 0.3$ & $3.9 \pm 0.8$ & $2.8 \pm 2.3$ & 8 & $3 b$ & 4 & 4 & $30.6 \pm 1.6$ & $33.4 \pm 1.7$ & $44.3 \pm 2.6$ & 70 \\
\hline $1 b$ & 54 & ND & $1.6 \pm 0.3$ & $3.7 \pm 0.7$ & $3.9 \pm 4.1$ & 7 & $3 b$ & 5 & 3 & $2.4 \pm 0.3$ & $6.1 \pm 0.8$ & $2.0 \pm 1.5$ & 92 \\
\hline $1 b$ & 55 & ND & $0.6 \pm 0.2$ & $1.5 \pm 0.5$ & $3.1 \pm 4.9$ & $<3$ & $3 b$ & 6 & 489 & $7.0 \pm 1.1$ & $84.1 \pm 2.6$ & $16.3 \pm 2.1$ & 64 \\
\hline $1 b$ & 56 & 1 & $5.0 \pm 0.6$ & $3.7 \pm 0.6$ & $7.0 \pm 3.6$ & 70 & $3 b$ & 15 & 90 & $6.7 \pm 1.0$ & $32.5 \pm 1.9$ & $4.8 \pm 2.9$ & 240 \\
\hline $1 b$ & 57 & ND & $76.0 \pm 6.1$ & $83.3 \pm 3.8$ & $96.3 \pm 23.2$ & 41 & $3 b$ & 46 & 2 & $31.1 \pm 1.8$ & $14.4 \pm 1.4$ & $31.1 \pm 6.5$ & 130 \\
\hline $1 b$ & 58 & ND & $15.4 \pm 0.7$ & $10.0 \pm 1.1$ & $17.0 \pm 4.7$ & 14 & $3 b$ & 87 & 9 & $14.4 \pm 1.0$ & $25.8 \pm 1.7$ & $20.3 \pm 5.5$ & 221 \\
\hline $1 b$ & 59 & ND & $23.0 \pm 1.5$ & $6.9 \pm 0.9$ & $23.5 \pm 3.6$ & 31 & $3 b$ & 88 & 1 & $9.6 \pm 0.8$ & $13.9 \pm 1.4$ & $6.9 \pm 2.4$ & 103 \\
\hline $1 b$ & 60 & ND & $8.2 \pm 0.6$ & $5.1 \pm 0.9$ & $9.4 \pm 1.3$ & 76 & $3 b$ & 91 & 593 & $10.4 \pm 1.0$ & $42.3 \pm 2.0$ & $13.9 \pm 4.5$ & 163 \\
\hline $1 b$ & 61 & 4 & $5.9 \pm 0.8$ & $1.3 \pm 0.9$ & $3.5 \pm 2.3$ & 66 & $3 b$ & 94 & ND & $12.3 \pm 1.2$ & $33.2 \pm 1.7$ & $25.3 \pm 8.1$ & 30 \\
\hline $1 b$ & 62 & ND & $5.0 \pm 0.7$ & $2.5 \pm 0.7$ & ND & 5 & $3 b$ & 98 & 107 & $16.7 \pm 0.8$ & $29.1 \pm 2.1$ & $6.8 \pm 2.5$ & 265 \\
\hline $1 b$ & 63 & ND & $13.4 \pm 1.1$ & $2.7 \pm 0.9$ & $7.7 \pm 3.9$ & 66 & $3 c$ & 100 & 4 & $7.7 \pm 0.9$ & $6.6 \pm 0.9$ & $5.5 \pm 3.1$ & 638 \\
\hline $1 b$ & 74 & 1 & $4.5 \pm 0.5$ & $3.2 \pm 0.8$ & $3.5 \pm 1.8$ & 80 & $3 c$ & 101 & 9 & $47.6 \pm 3.4$ & $56.5 \pm 2.8$ & $68.4 \pm 17.7$ & 117 \\
\hline $1 b$ & 75 & 1 & $19.4 \pm 1.2$ & $19.3 \pm 1.2$ & $41.3 \pm 7.5$ & 14 & $3 c$ & 102 & 348 & $103.4 \pm 6.5$ & $184.3 \pm 5.8$ & $132.7 \pm 26.7$ & 156 \\
\hline 2 & 11 & ND & $5.9 \pm 0.4$ & $8.7 \pm 1.7$ & $6.3 \pm 1.4$ & 5 & $3 c$ & 103 & ND & $4.7 \pm 0.4$ & $7.1 \pm 1.3$ & $4.5 \pm 3.2$ & 364 \\
\hline 2 & 64 & ND & $71.4 \pm 6.2$ & $68.3 \pm 2.3$ & $83.1 \pm 23.5$ & 13 & $3 c$ & 104 & 97 & $164.9 \pm 8.5$ & $129.1 \pm 4.8$ & $199.6 \pm 34.8$ & $227^{\mathrm{b}}$ \\
\hline 2 & 65 & ND & $8.4 \pm 0.7$ & $18.9 \pm 1.6$ & $14.6 \pm 4.5$ & 25 & $3 c$ & 105 & 174 & $4.6 \pm 0.4$ & $1.4 \pm 1.1$ & $6.1 \pm 6.6$ & 358 \\
\hline 2 & 66 & ND & $9.6 \pm 0.7$ & $10.2 \pm 1.2$ & $5.2 \pm 3.8$ & 7 & $3 c$ & 106 & 4 & $14.5 \pm 2.0$ & $31.4 \pm 2.0$ & $15.3 \pm 7.4$ & 380 \\
\hline 2 & 67 & 1 & $1.5 \pm 0.2$ & $3.4 \pm 0.6$ & $1.9 \pm 1.6$ & 25 & $3 c$ & 107 & 21 & $4.1 \pm 0.5$ & $6.1 \pm 0.9$ & $3.2 \pm 2.5$ & 188 \\
\hline 2 & 68 & ND & $6.4 \pm 0.8$ & $2.9 \pm 0.8$ & $5.5 \pm 1.5$ & 24 & $3 c$ & 108 & 34 & $74.8 \pm 5.2$ & $69.7 \pm 3.5$ & $75.8 \pm 17.7$ & 223 \\
\hline 2 & 69 & ND & $16.3 \pm 1.2$ & $21.0 \pm 1.5$ & $17.3 \pm 5.6$ & 57 & $3 c$ & 109 & 2 & $9.1 \pm 1.9$ & $13.0 \pm 1.3$ & $5.5 \pm 4.0$ & 594 \\
\hline 2 & 70 & ND & $5.6 \pm 0.8$ & $10.3 \pm 1.1$ & $10.1 \pm 2.3$ & 79 & $3 c$ & 110 & ND & $22.5 \pm 1.7$ & $18.0 \pm 1.5$ & $17.1 \pm 6.1$ & 135 \\
\hline 2 & 71 & ND & $4.1 \pm 0.5$ & $4.8 \pm 0.9$ & $10.0 \pm 4.4$ & $<3$ & $3 c$ & 111 & 2 & $11.6 \pm 1.0$ & $12.0 \pm 1.3$ & $9.6 \pm 3.4$ & 187 \\
\hline 2 & 72 & ND & $14.8 \pm 0.9$ & $7.0 \pm 0.9$ & $13.2 \pm 3.1$ & 42 & $3 c$ & 112 & ND & $13.1 \pm 1.1$ & $14.6 \pm 1.4$ & $18.9 \pm 5.9$ & 695 \\
\hline 2 & 73 & ND & $3.2 \pm 0.5$ & $2.7 \pm 0.6$ & ND & 100 & $3 c$ & 113 & 4 & $16.8 \pm 1.3$ & $16.7 \pm 1.6$ & $11.8 \pm 4.2$ & 411 \\
\hline 2 & 76 & ND & $1.7 \pm 0.2$ & $2.1 \pm 0.5$ & $2.7 \pm 3.1$ & 5 & $3 c$ & 114 & 2 & $7.9 \pm 0.9$ & $16.7 \pm 1.4$ & $7.6 \pm 3.5$ & 471 \\
\hline 2 & 77 & ND & $10.8 \pm 0.7$ & $16.7 \pm 1.4$ & $9.8 \pm 1.9$ & 97 & $3 c$ & 115 & 36 & $4.2 \pm 0.6$ & $6.5 \pm 1.1$ & $3.1 \pm 2.4$ & 548 \\
\hline 2 & 78 & ND & $3.6 \pm 0.4$ & $5.3 \pm 0.9$ & $6.3 \pm 1.6$ & 90 & $3 c$ & 116 & 9 & $4.4 \pm 1.1$ & $13.6 \pm 1.1$ & $2.7 \pm 3.0$ & 643 \\
\hline 2 & 79 & ND & $0.7 \pm 0.2$ & $0.9 \pm 0.6$ & $1.1 \pm 3.2$ & 51 & $3 c$ & 117 & 1 & $6.3 \pm 0.5$ & $8.1 \pm 1.2$ & $8.2 \pm 2.7$ & 78 \\
\hline 2 & 80 & ND & $4.0 \pm 0.4$ & $6.0 \pm 1.2$ & $4.4 \pm 2.5$ & 67 & & & & & & & \\
\hline
\end{tabular}

Errors are $\pm 2 \sigma$ from counting statistics. ND indicates not detected; these were treated as zero activities in data analysis.

a Values less than the reporting limit of $3 \mathrm{~Bq} \mathrm{~L}^{-1}$ were treated as zero values for data analysis.

b Rn sample collected on a later date than indicated on Table 2.

metasedimentary and metavolcanic rocks (Table 3 ). Within the Rolesville Granite, the median ${ }^{228} \mathrm{Ra} /{ }^{226} \mathrm{Ra}$ of groundwater is 0.68 (Table 3 ), but the median $\mathrm{Th} / \mathrm{U}$ activity ratio calculated from Th and $\mathrm{U}$ content of Rolesville Granite rock samples (median $17.2 \mathrm{mg} \mathrm{kg}^{-1}$ and $4.4 \mathrm{mg} \mathrm{kg}^{-1}$, respectively) is 1.2 ( $n=42$; data from Costain et al., 1977; Speer and Hoff, 1997; McSwain et al., in review). These radionuclide levels are not unusually high for granites (e.g. Gascoyne, 1989; Speer and Hoff, 1997). In addition, the median ${ }^{228} \mathrm{Ra} /{ }^{226} \mathrm{Ra}$ of water samples is lower than the median ${ }^{228} \mathrm{Ra} /{ }^{226} \mathrm{Ra}$ ratio measured directly from Rolesville Granite bulk rock samples (1.36; Table 5). These ratios imply that some excess ${ }^{226}$ Ra relative to ${ }^{228} \mathrm{Ra}$ is available to fracture surfaces, compared to bulk rock ratios. Three possible mechanisms could reduce ${ }^{228} \mathrm{Ra} /{ }^{226} \mathrm{Ra}$ in water relative to the bulk rock: (1) relative ${ }^{226} \mathrm{Ra}$ enrichment could be derived from preferential recoil processes within each decay series, enhancing the mobility of successive decay products. ${ }^{226} \mathrm{Ra}$ is the third alpha decay in its decay series, but ${ }^{228} \mathrm{Ra}$ is the first decay step and ${ }^{224} \mathrm{Ra}$ is the second in the ${ }^{232} \mathrm{Th}$ decay series. Thus, ${ }^{226} \mathrm{Ra}$ would be more available due to the recoil effect placing it closer to the mineral surface (Davidson and Dickson, 1986); (2) U, being more soluble than Th, could be mobilized by weathering or the groundwater system to 
Table 5

Radium isotope activities in Rolesville Granite samples from cores WC- 1 and WC-3

\begin{tabular}{llll}
\hline Sample & ${ }^{226} \mathrm{Ra}\left(\mathrm{Bq} \mathrm{kg}{ }^{-1}\right)$ & ${ }^{228} \mathrm{Ra}\left(\mathrm{Bq} \mathrm{kg}{ }^{-1}\right)$ & ${ }^{228} \mathrm{Ra} /{ }^{226} \mathrm{Ra}$ \\
\hline WC-1-41.4 & $43.0 \pm 5.6$ & $42.4 \pm 4.6$ & 0.99 \\
WC-1-59.6 & $31.3 \pm 3.8$ & $61.5 \pm 3.8$ & 1.97 \\
Duplicate can & $27.3 \pm 2.5$ & $53.3 \pm 2.5$ & 1.95 \\
WC-1-70.0 & $53.5 \pm 5.6$ & $56.5 \pm 5.0$ & 1.06 \\
Duplicate can & $51.9 \pm 5.7$ & $54.8 \pm 5.2$ & 1.06 \\
WC-3-48.5 & $44.6 \pm 4.4$ & $72.1 \pm 4.2$ & 1.62 \\
WC-3-66.3 & $20.8 \pm 5.8$ & $55.6 \pm 5.8$ & 2.68 \\
WC-3-117.3 & $47.8 \pm 5.9$ & $51.9 \pm 4.9$ & 1.09 \\
\hline
\end{tabular}

Errors are $\pm 2 \sigma$ from counting statistics.

provide additional ${ }^{226} \mathrm{Ra}$ precursors to adsorption sites (e.g. Michel, 1984). Although some fractionation of ${ }^{228} \mathrm{Ra} /{ }^{226} \mathrm{Ra}$ is observed relative to bulk rock samples, we note no predominance of ${ }^{226} \mathrm{Ra}$ that would indicate major net accumulations of $U$ on fracture surfaces because wells with elevated overall Ra tend to contain both ${ }^{228} \mathrm{Ra}$ and ${ }^{226} \mathrm{Ra}$. Localized minerals containing both $\mathrm{U}$ and $\mathrm{Th}$ and/or adjacent adsorption sites in fractures may be necessary, which implies that primary and/or secondary minerals are significant Ra sources rather than net additions from long-range $U$ transport in groundwater; and (3) dissolution of

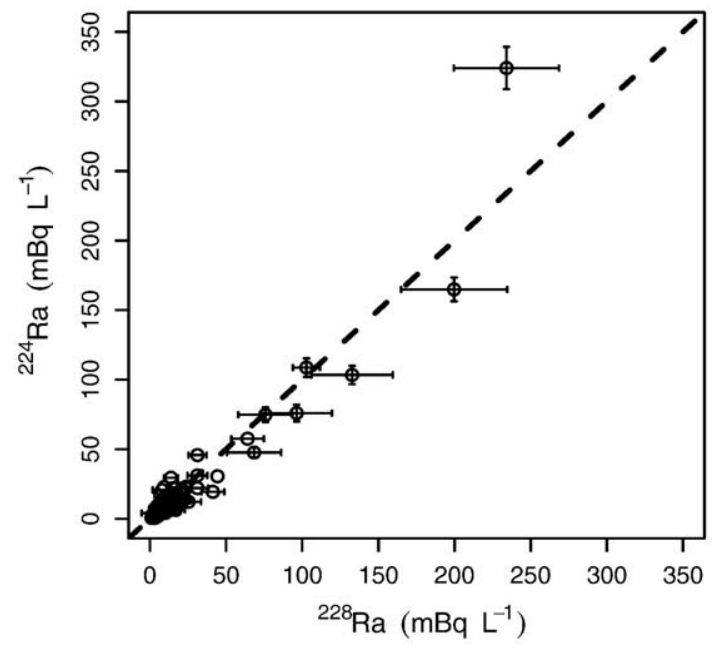

Fig. 5. ${ }^{228} \mathrm{Ra}$ and ${ }^{224} \mathrm{Ra}$ activities in groundwater. Dashed line represents equilibrium value of 1 . Counting error omitted where smaller than symbol size. aquifer minerals would generate lower ${ }^{228} \mathrm{Ra} /{ }^{226} \mathrm{Ra}$ due to the half-life difference between ${ }^{228} \mathrm{Ra}$ and ${ }^{226} \mathrm{Ra}$ and the slow rate of dissolution (Hammond et al., 1988). Dissolution as a direct source of Ra is ruled out given that short-lived ${ }^{224} \mathrm{Ra}$ is present at similar activities as ${ }^{228} \mathrm{Ra}$ (Fig. 5; Section 4.2.2), indicating rapid local interaction with the aquifer rocks. Also, the strong retardation of Ra in fresh waters (retardation factor $\sim 10^{4}-10^{5}$; Krishnaswami et al., 1982; Dickson, 1990) would prevent ${ }^{226} \mathrm{Ra}$ from accumulating along a flowpath relative to ${ }^{228} \mathrm{Ra}$.

\subsection{2. ${ }^{224} \mathrm{Ra} /{ }^{228} \mathrm{Ra}$ activity ratio}

Because all isotopes of Ra exhibit identical chemical behavior but are generated by recoil at different rates, short-lived to long-lived isotope ratios may indicate the rates of Ra contribution or removal. High ratios may indicate that the effective first-order rate constant of Ra immobilization exceeds the ingrowth rate (decay constant) of longlived Ra from alpha recoil, thus indicating that Ra sinks are effective (Krishnaswami et al., 1982; Martin and Akber, 1999; Moise et al., 2000). Low short-lived to long-lived isotope ratios may indicate a Ra source from primary mineral dissolution in which short-lived Ra decays faster than the dissolution rate (Hammond et al., 1988). On average, ${ }^{224} \mathrm{Ra} /{ }^{228} \mathrm{Ra}$ activity ratios are close to the expected equilibrium value of 1 (Fig. 5; Table 3). This probably indicates that (1) Ra sources are broadly distributed on fracture surfaces or are at least very close to the sampling point, rather than being mobilized in an earlier phase of groundwater evolution that would allow significant ${ }^{224} \mathrm{Ra}$ decay; and (2) the rates of critical Ra-mobilizing processes do not generate large changes in ${ }^{224} \mathrm{Ra} /{ }^{228} \mathrm{Ra}$. Thus, we conclude that $\mathrm{Ra}$ is not released significantly by mineral dissolution, and the rate constant of Ra adsorption allows long- and short-lived isotopes to be generated by recoil without large fluctuations in Ra isotope ratios.

\subsubsection{Radon-222}

Radon-222 activity in water may be interpreted as recoil flux from mineral surfaces (Krishnaswami et al., 1982) or more complexly as a combination of recoil and diffusion of Rn from microfractures resulting from a higher effective surface area for Rn relative to Ra (Rama and Moore, 1984; Davidson and Dickson, 1986; Andrews et al., 1989). In this study, ${ }^{222} \mathrm{Rn}$ is strongly associated with rock type, and in particular, groundwater from the Rolesville Granite exhibits approximately one order of magnitude higher median ${ }^{222} \mathrm{Rn}$ (Table 3). In the granite, ${ }^{222} \mathrm{Rn}$ activity is positively correlated with closed casing depth $(\rho=0.61)$, somewhat correlated with DO ( $\rho=0.42$; Fig. 4$)$, and negatively correlated
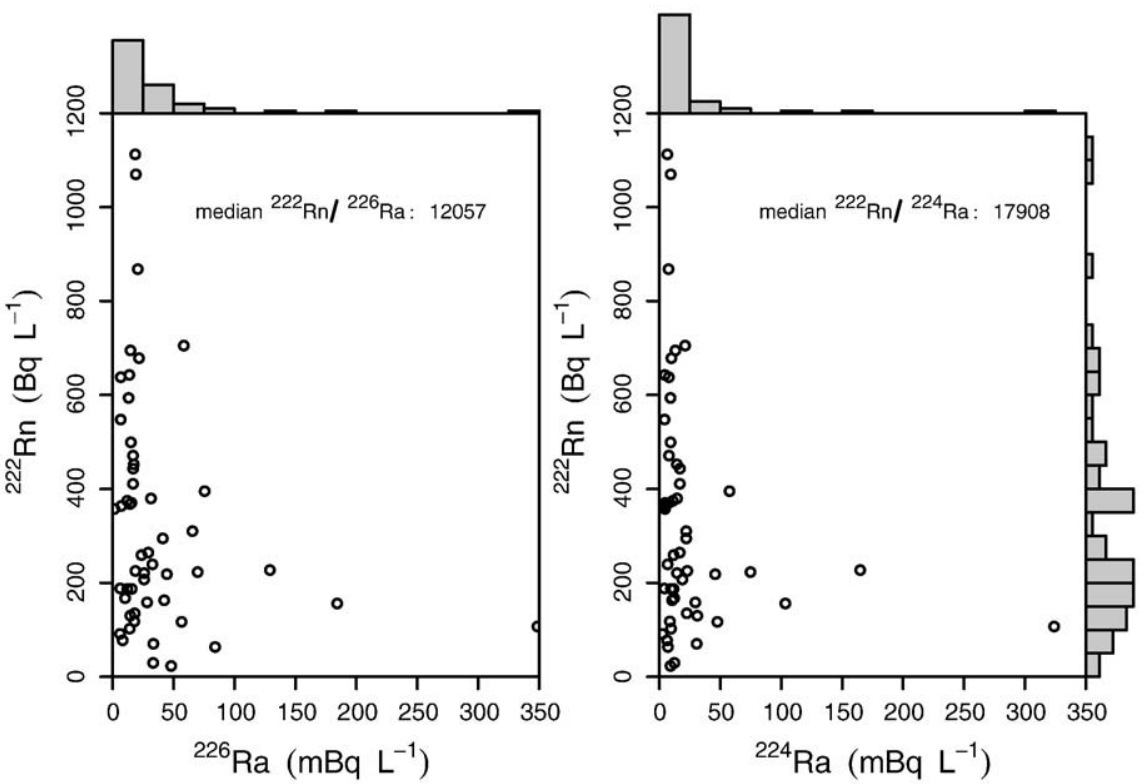

Fig. 6. Relationship between ${ }^{222} \mathrm{Rn}$ and ${ }^{226} \mathrm{Ra}$ (left), ${ }^{222} \mathrm{Rn}$ and ${ }^{224} \mathrm{Ra}$ (right) and histograms of isotope activities in Rolesville Granite groundwater. 

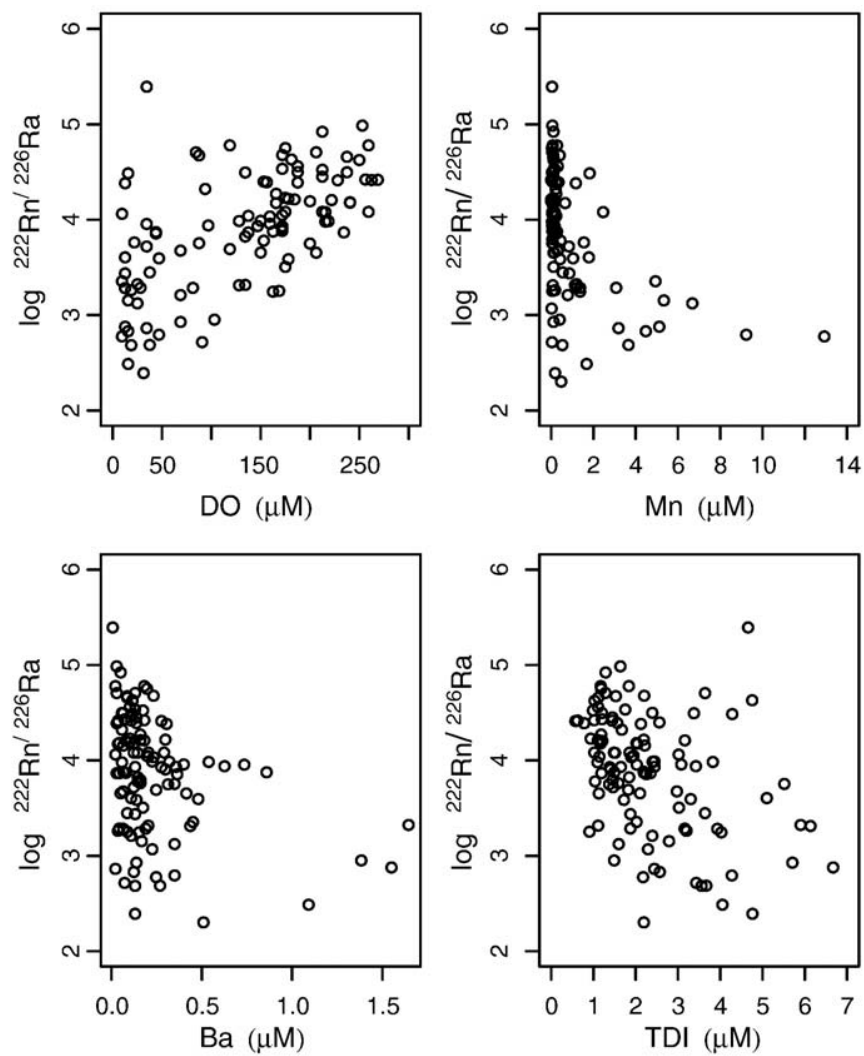

Fig. 7. Relationship between dissolved oxygen and Mn (top), Ba and total ions (bottom), and the ${ }^{222} \mathrm{Rn} /{ }^{226} \mathrm{Ra}$ activity ratio.

to some degree with most major cations including $\mathrm{Ba}(\rho=-0.40)$. Overall, high Rn occurs mainly in oxic, low-TDS waters (Fig. 4). As Rn is inert, these relationships indicate (1) that more oxic, low-TDS conditions support more adsorbed Ra on fracture surfaces per volume of water, consistent with a recoil-dominated interpretation (Krishnaswami et al., 1982); or (2) that oxic fractures contain a larger surface area per volume of water, consistent with a diffusion-based interpretation (Rama and Moore, 1984).

\subsection{4. ${ }^{222} \mathrm{Rn} /{ }^{226} \mathrm{Ra}$ activity ratio}

${ }^{222} \mathrm{Rn} /{ }^{226} \mathrm{Ra}$ ratio distributions are generally consistent between rock types (Table 3 ), suggesting that ${ }^{222} \mathrm{Rn} /{ }^{226} \mathrm{Ra}$ provides information on Ra adsorption in crystalline rock fractures regardless of the Ra and $\mathrm{Rn}$ sources in each rock type. If the assumption that ${ }^{222} \mathrm{Rn}$ activity in the water is proportional to the recoil flux of Rn and Ra from the aquifer rocks is used, then the $\mathrm{Rn} / \mathrm{Ra}$ activity ratio is roughly equivalent to the distribution coefficient $K_{\mathrm{D}}$ for radium (i.e., adsorption-desorption equilibrium between aqueous and adsorbed Ra; Krishnaswami et al., 1982; Dickson, 1990). The highest activities of $\mathrm{Ra}$ and $\mathrm{Rn}$ occur in distinct samples and thus $\mathrm{Rn} / \mathrm{Ra}$ occupies a large range. Given the approximately lognormal distributions of $\mathrm{Rn}$ and $\mathrm{Ra}$, median values of ${ }^{222} \mathrm{Rn} /{ }^{226} \mathrm{Ra}$ and ${ }^{222} \mathrm{Rn} /{ }^{224} \mathrm{Ra}$ on the order of $10^{4}$ approximate $K_{\mathrm{D}}$ (Fig. 6). Among redox-sensitive solutes, dissolved oxygen concentration is positively correlated with ${ }^{222} \mathrm{Rn} /{ }^{226} \mathrm{Ra}$ $(\rho=0.52$; Fig. 7$)$, and $\mathrm{Mn}$ is negatively correlated with ${ }^{222} \mathrm{Rn} /{ }^{226} \mathrm{Ra}$ $\left(\rho=-0.56\right.$; Fig. 7). Also, ${ }^{222} \mathrm{Rn} /{ }^{226} \mathrm{Ra}$ exhibits correlation with alkaline earth metals ( $\rho=-0.48$ for $\mathrm{Ba},-0.47$ for $\mathrm{Ca},-0.45$ for $\mathrm{Sr}$, and -0.41 for TDI). As Ba in particular may compete with Ra for adsorption sites, these trends could indicate that adsorption of Ra is related to cation concentrations, probably derived from the weathering zone. Similar correlations are evident if ${ }^{222} \mathrm{Rn} /{ }^{224} \mathrm{Ra}$ is used to represent apparent $K_{D}$ instead of ${ }^{222} \mathrm{Rn} /{ }^{226} \mathrm{Ra}$. The significance of this approach is that the similar half-lives of ${ }^{222} \mathrm{Rn}$ and ${ }^{224} \mathrm{Ra}$ (3.8 d and $3.7 \mathrm{~d}$, respectively) negate dissolution contributions of long-lived ${ }^{226} \mathrm{Ra}$ (Krishnaswami et al., 1982). The median apparent $K_{\mathrm{D}}$ estimated from ${ }^{222} \mathrm{Rn} /{ }^{226} \mathrm{Ra}$ decreases by about one order of magnitude between oxygen-saturated and anoxic conditions and along the range of $\mathrm{Mn}$, TDI, and $\mathrm{Ba}$ concentrations ( 20,000 to $\sim 2000$; Fig. 8). In summary, trends in ${ }^{222} \mathrm{Rn} /{ }^{226} \mathrm{Ra}$ suggest that adsorption of recoil-produced Ra varies as a result of local chemical conditions.

\subsection{Overview of radionuclide sources and sinks}

At small scales, much of the $U$ in granite is distributed in secondary minerals in microfractures and adsorbed to Fe oxides, Mn oxides, and clays (Speer et al., 1981; Guthrie and Kleeman, 1986; Wathen, 1987). In addition, significant disequilibrium of solid-phase $U$ - and Th-series radionuclides at the scale of meters indicates long-term mobility of radionuclides, probably by groundwater (e.g. Michel, 1984). Long-lived $\mathrm{U}$ may be mobile in groundwater systems until reaching reducing zones, at which it is strongly adsorbed to Fe oxides (Ames et al., 1983; Waite et al., 1994). However, the strong positive correlation of ${ }^{226} \mathrm{Ra}$ and ${ }^{228} \mathrm{Ra}$ obtained in this study indicates that both ${ }^{226} \mathrm{Ra}$ and ${ }^{228} \mathrm{Ra}$ are present within recoil range of fracture surfaces, indicating the cooccurrence of Th and $U$ in primary and/or secondary minerals near fracture surfaces.

The relatively high minimum values of apparent $K_{\mathrm{D}}$ in fractured crystalline rocks $\left(\sim 10^{3}\right)$ indicate that Ra is rapidly adsorbed in fresh waters in bedrock fractures to balance input from alpha recoil, and that Ra does not exhibit the lower apparent $K_{\mathrm{D}}$ commonly observed in
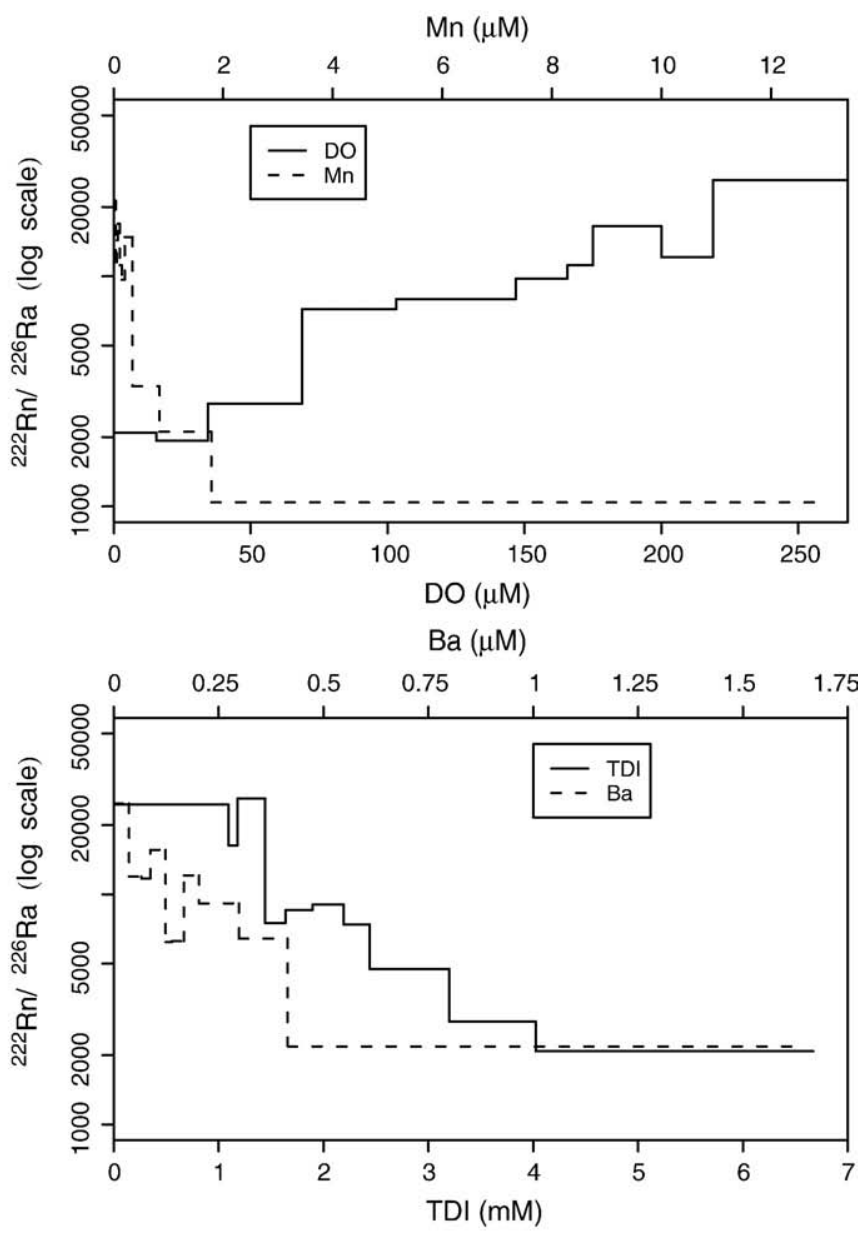

Fig. 8. Apparent $K_{\mathrm{D}}$ across dissolved oxygen and $\mathrm{Mn}$ (top) and Ba and total ions (bottom). Steps represent median ${ }^{222} \mathrm{Rn} /{ }^{226} \mathrm{Ra}$ in each of 10 concentration intervals containing approximately equal numbers of samples. 
sandstone aquifers $\left(\sim 10^{1}-10^{3}\right.$; King et al., 1982; Lively et al., 1992; Reynolds et al., 2003) or saline aquifers $\left(\sim 10^{0}-10^{2}\right.$; Sturchio et al., 2001). Net adsorption of Ra is less effective under the low-DO, higherTDS conditions observed in this study because (1) anoxic fractures contain lower surface area of redox-sensitive Ra adsorption sites, such as Mn oxides; and/or (2) higher concentrations of alkaline earth metals, such as Ba, compete with recoil-generated Ra for adsorption sites. The down-flow transition from the saprolite to water-producing bedrock fractures involves increasing water-to-mineral surface ratio, decreasing clay content, and decreasing mineral-water contact time. Thus, cations in waters passing from highly to lightly weathered zones may experience increased competition for adsorption sites. Our results indicate that in the saprolite-fractured crystalline rock system, major ion concentrations and redox state (dissolved oxygen consumption, Mn oxide reduction, and Fe oxide reduction) are linked. Fractures receiving water from more weathered recharge sources contain higher concentrations of alkaline earth metals and bicarbonate. As these waters also tend to exhibit lower DO concentrations, they have also been more involved in organic carbon oxidation and/or biotite and pyrite oxidation. Consequently, the distinction between the individual contributions of cation concentrations versus redox conditions on Ra mobilization is not straightforward, and we cannot differentiate the redox and cation exchange effects on apparent $K_{\mathrm{D}}$.

\section{Conclusions}

Although the main influence on radionuclides in groundwater is the $U$ and Th content on fracture surfaces, linkage between weathering-derived ion concentrations, redox conditions, and radionuclide mobility in bedrock fractures occurs across a range of rock types and radionuclide activities. The primary source of Ra and $\mathrm{Rn}$ in groundwater is recoil from parent radionuclides on fracture surfaces, which is balanced by adsorption of Ra. While groundwater chemistry is mostly acquired during recharge through the soil and saprolite, radionuclides are generated by recoil in the saturated bedrock fracture network. Thus, the efficiency of adsorption of Ra is influenced by the redoxsensitive stability of adsorption sites and/or competition with other divalent cations. In low-DO samples, elevated Mn and Fe concentrations suggest that their oxides are subject to reductive dissolution and are thus less efficient at removing Ra. This interpretation is consistent with observed trends in Ra and Rn activities, as well as variable ${ }^{222} \mathrm{Rn} /$ ${ }^{226} \mathrm{Ra}$ ratios correlated with $\mathrm{DO}, \mathrm{Mn}, \mathrm{Ba}$, and TDI concentrations. Overall, the approximately one order of magnitude variation in the apparent Ra distribution coefficient across the range of dissolved oxygen and alkaline earth metal concentrations indicates that in fresh groundwaters from fractured crystalline rocks, water chemistry is an important influence of on Ra adsorption, although secondary in importance to the radionuclide content of the solids.

\section{Acknowledgments}

Well sampling was arranged by Lori Bennear, Elizabeth Pratson, and Soumya Balasubramanya. We thank Hadas Raanan, Nick Baker, Timothy Foley, Scott Geis, Nicole Hagan, Waruntorn Kanitpanyacharoen, James Leeper, Anna Grzebien, and Kerry Schlichting for field and laboratory assistance, and Paul Heine, Paul Baker, and Emily Klein for lab access and assistance. We also thank Phil Bradley and Michael Medina of the North Carolina Geological Survey; Douglas Harned, Melinda Chapman, and Kristen McSwain of the U.S. Geological Survey; and Richard Bolich of the North Carolina Division of Water Quality for providing unpublished data and core samples; and Joshua Tootoo of Duke University Children's Environmental Health Initiative for GIS assistance. Helpful discussions with W.S. Moore and Ralph Arnold improved the research leading to this manuscript. This work was supported by U.S. Department of Agriculture (USDA) Cooperative State Research Education and Extension Service (CSREES) grant NCW-2006-03956 and the Nicholas School of the
Environment at Duke University. We also thank two anonymous reviewers for their constructive comments and Guest Editors Jennifer Roberts and Annette Summers Engel for editorial handling.

\section{References}

Ames, L.L., McGarrah, J.E., Walker, B.A., Salter, P.F., 1983. Uranium and radium sorption on amorphous ferric oxyhydroxide. Chemical Geology 40, 135-148.

Andrews, J.N., Ford, D.J., Hussain, N., Trivedi, D., Youngman, M.J., 1989. Natural radioelement solution by circulating groundwaters in the Stripa granite. Geochimica et Cosmochimica Acta 53, 1791-1802.

Asikainen, M., 1981a. Radium content and the Ra-226/Ra-228 activity ratio in groundwater from bedrock. Geochimica et Cosmochimica Acta 45, 1375-1381.

Asikainen, M., 1981b. State of disequilibrium between U-238, U-234, Ra-226, and Rn222 in groundwater from bedrock. Geochimica et Cosmochimica Acta 45, 201-206.

Asikainen, M., Kahlos, H., 1979. Anomalously high concentrations of uranium, radium and radon in water from drilled wells in the Helsinki region. Geochimica et Cosmochimica Acta 43, 1681-1686.

Banwart, S., Tullborg, E., Pedersen, K., Gustafsson, E., Laaksoharju, M., Nilsson, A., Wallin, B., Wikberg, P., 1996. Organic carbon oxidation induced by large-scale shallow water intrusion into a vertical fracture zone at the Aspo Hard Rock Laboratory (Sweden). Journal of Contaminant Hydrology 21, 115-125.

Banwart, S.A., Wikberg, P., Puigdomenech, I., 1999. Protecting the redox stability of a deep repository: Concepts, results, and experience from the Aspo hard rock laboratory. In: Metcalfe, R., Rochelle, C.A. (Eds.), Chemical containment of waste in the geosphere Special Publication - Geological Society of London, vol. 157, pp. 85-99.

Bolton, D.W., 2000. Occurrence and distribution of radium, gross alpha-particle activity, and gross-beta particle activity in ground water in the Magothy Formation and Potomac Group aquifers, upper Chesapeake Bay area, Maryland. Maryland Geological Survey, Report of Investigations, vol. 70.

Buesseler, K.O., Andrews, J.A., Hartman, M.C., Belastock, R., Chai, F., 1995. Regional estimates of the export flux of particulate organic carbon derived from thorium-234 during the JGOFS EqPac program. Deep Sea Research Part II: Topical Studies in Oceanography 42, 777-791.

Cecil, L.D., Smith, R.C., Reilly, M.A., Rose, A.W., 1987. Radium-228 and radium-226 in ground water of the Chickies Formation, southeastern Pennsylvania. In: Graves, B. (Ed.), Radon, Radium, and Other Radioactivity in Ground Water. Lewis Publishers, Chelsea, MI, pp. 437-447.

Clark, T.W., Blake, D.E., Stoddard, E.F., Carpenter, P.A., Carpenter, R.H., 2004. Preliminary bedrock geologic map of the Raleigh $30^{\prime} \times 60^{\prime}$ quadrangle, North Carolina. North Carolina Geological Survey, Open-File Report 2004-02.

Costain, J.K., Perry, L.D., Dunbar, J.A., 1977. Geothermal gradients, heat flow, and heat generation. In: Costain, J.K., Glover, L., Sinha, A.K. (Eds.), Evaluation and targeting of geothermal energy resources in the southeastern United States. Report to U.S. Department of Energy VPI-SU-5648-1, C28-C45.

Daniel, C.C., Dahlen, P.R., 2002. Preliminary hydrogeologic assessment and study plan for a regional ground-water resource investigation of the Blue Ridge and Piedmont Provinces of North Carolina. U.S. Geological Survey, Water-Resources Investigations Report 02-4105.

Davidson, M.R., Dickson, B.L., 1986. A porous flow model for steady state transport of radium in groundwater. Water Resources Research 22, 34-44.

Dickson, B.L., 1990. Radium in groundwater. The Environmental Behaviour of Radium, vol.1. International Atomic Energy Agency, Technical Reports Series, vol. 310, pp. 335-372.

Dickson, B.L., Herczeg, A.L., 1992. Naturally-occurring radionuclides in acid-saline groundwaters around Lake Tyrrell, Victoria, Australia. Chemical Geology 96, 95-114

Drever, J.I., 1997. Geochemistry of natural waters: Surface and groundwater environments, 3rd ed. Prentice-Hall, Upper Saddle River, NJ.

Environmental Protection Agency, 2000. National Primary Drinking Water Regulations; Radionuclides; Final Rule. Federal Register 65, 76708-76753.

Garcia-Solsona, E., Garcia-Orellana, J., Masqué, P., Dulaiova, H., 2008. Uncertainties associated with ${ }^{223} \mathrm{Ra}$ and ${ }^{224} \mathrm{Ra}$ measurements in water via a Delayed Coincidence Counter (RaDeCC). Marine Chemistry 109, 198-219.

Gascoyne, M., 1989. High levels of uranium and radium in groundwaters at Canada's Underground Research Laboratory, Lac du Bonnet, Manitoba, Canada. Applied Geochemistry 4, 577-591.

Gascoyne, M., 1997. Evolution of redox conditions and groundwater composition in recharge-discharge environments on the Canadian Shield. Hydrogeology Journal 5(3), 4-18.

Gascoyne, M., 2004. Hydrogeochemistry, groundwater ages and sources of salts in a granitic batholith on the Canadian Shield, southeastern Manitoba. Applied Geochemistry 19 519-560.

Gilkeson, R.H., Perry, E.C., Cowart, J.B., Holtzman, R.B., 1984. Isotopic studies of the natural sources of radium in groundwater in Illinois. Illinois Water Resources Center, Research Report, vol. 187.

Grundl, T., Cape, M., 2006. Geochemical factors controlling radium activity in a sandstone aquifer. Ground Water 44, 518-527.

Guthrie, V.A., Kleeman, J.D., 1986. Changing uranium distributions during weathering of granite. Chemical Geology 54, 113-126.

Hammond, D.E., Zukin, J.G., Ku, T., 1988. The kinetics of radioisotope exchange between brine and rock in a geothermal system. Journal of Geophysical Research 93, 13175-13186.

Herczeg, A.L., Simpson, H.J., Anderson, R.F., Trier, R.M., Mathieu, G.G., Deck, B.L., 1988 Uranium and radium mobility in groundwaters and brines within the Delaware Basin, Southeastern New Mexico, U.S.A. Chemical Geology, Isotope Geoscience Section 72, 181-196 
Herranz, M., Idoeta, R., Abelairas, A., Legarda, F., 2006. Radon fixation for determination of ${ }^{224} \mathrm{Ra},{ }^{226} \mathrm{Ra}$ and ${ }^{228} \mathrm{Ra}$ via gamma-ray spectrometry. Radiation Measurements 41 486-491.

Ivanovich, M., Murray, A., 1992. Spectroscopic methods, In: Ivanovich, M., Harmon, R.S (Eds.), Uranium-series Disequilibrium: Applications to Earth, Marine, and Environmental Sciences, 2nd ed. Oxford University Press, Oxford, pp. 127-173.

King, P.T., Michel, J., Moore, W.S., 1982. Ground water geochemistry of ${ }^{228} \mathrm{Ra},{ }^{226} \mathrm{Ra}$ and ${ }^{222} \mathrm{Rn}$. Geochimica et Cosmochimica Acta 46, 1173-1182.

Kosecki, J., Fodor, R.V., 1997. Petrology, mineralogy, and geochemistry of the Rolesville granitic batholith, eastern Piedmont, North Carolina. Southeastern Geology 37, 91-107.

Kraemer, T.F., Reid, D.F., 1984. The occurrence and behavior of radium in saline formation water of the U.S. Gulf Coast region. Isotope Geoscience 2, 153-174.

Krishnaswami, S., Graustein, W.C., Turekian, K.K., Dowd, J.F., 1982. Radium, thorium, and radioactive lead isotopes in groundwaters: application to the in situ determination of adsorption-desorption rate constants and retardation factors. Water Resources Research 18,1663-1675.

Landa, E.R., Phillips, E.J.P., Lovley, D.R., 1991. Release of ${ }^{226}$ Ra from uranium mill tailings by microbial Fe(III) reduction. Applied Geochemistry 6, 647-652.

Langmuir, D., Riese, A.C., 1985. The thermodynamic properties of radium. Geochimica et Cosmochimica Acta 49, 1593-1601.

Lively, R.S., Jameson, R., Alexander, E.C., Morey, G.B., 1992. Radium in the Mt. SimonHinckley aquifer, east-central and southeastern Minnesota. Minnesota Geological Survey, Information Circular, vol. 36.

Luo, S., Ku, T., Roback, R., Murrell, M., McLing, T., 2000. In-situ radionuclide transport and preferential groundwater flows at INEEL (Idaho): decay-series disequilibrium studies. Geochimica et Cosmochimica Acta 64, 867-881.

Martin, P., Akber, R.A., 1999. Radium isotopes as indicators of adsorption-desorption interactions and barite formation in groundwater. Journal of Environmental Radioactivity $46,271-286$.

Martin, A.J., Crusius, J., Jay McNee, J., Yanful, E.K., 2003. The mobility of radium-226 and trace metals in pre-oxidized subaqueous uranium mill tailings. Applied Geochemistry $18,1095-1110$.

Mays, C.W., Rowland, R.E., Stehney, A.F., 1985. Cancer risk from the lifetime intake of Ra and U isotopes. Health Physics 48, 635-647.

McSwain, K.B., Chapman, M.J., Huffman, B.A., and Bolich, R.E., in review. Waterresources data and hydrogeologic setting at the Raleigh Hydrogeologic Research Station, Wake County, North Carolina. U.S. Geological Survey, Open-File Report.

Michel, J., 1984. Redistribution of uranium and thorium series isotopes during isovolumetric weathering of granite. Geochimica et Cosmochimica Acta 48, 1249-1255.

Moise, T., Starinsky, A., Katz, A., Kolodny, Y., 2000. Ra isotopes and Rn in brines and ground waters of the Jordan-Dead Sea Rift Valley: enrichment, retardation, and mixing. Geochimica et Cosmochimica Acta 64, 2371-2388.

Moon, D.S., Burnett, W.C., Nour, S., Horwitz, P., Bond, A, 2003. Preconcentration of radium isotopes from natural waters using $\mathrm{MnO}_{2}$ Resin. Applied Radiation and Isotopes 29, 255-262.

Moore, W.S., Arnold, R., 1996. Measurement of ${ }^{223}$ Ra and ${ }^{224}$ Ra in coastal waters using a delayed coincidence counter. Journal of Geophysical Research C: Oceans 101,1321-1329.

Moore, W.S., Reid, D.F., 1973. Extraction of radium from natural water using manganese impregnated acrylic fibers. Journal of Geophysical Research 78, 8880-8886.

National Research Council, 1999. Risk Assessment of Radon in Drinking Water. Nationa Academies Press, Washington.

Nelms, D.L., Harlow, G.E., 2003. Aquifer susceptibility in Virginia: data on chemical and isotopic composition, recharge temperature, and apparent age of water from wells and springs, 1998-2000. U.S. Geological Survey, Open-File Report 2003-246.

Nirdosh, I., Trembley, W.B., Johnson, C.R., 1990. Adsorption-desorption studies on the ${ }^{226}$ Ra-hydrated metal oxide systems. Hydrometallurgy 24, 237-248.

Parkhurst, D.L., Appelo, C.A.J., 1999. User's guide to PHREEOC (version 2) - a computer program for speciation, batch-reaction, one-dimensional transport, and inverse geochemical calculations. U.S. Geological Survey, Water-Resources Investigations Report 99-4259.

Phillips, E.J.P., Landa, E.R., Kraemer, T, Zielinski, R., 2001. Sulfate-reducing bacteria release barium and radium from naturally occurring radioactive material in oil-field barite. Geomicrobiology Journal 18, 167-182.
Porcelli, D., 2008. Investigating groundwater processes using U- and Th-series nuclides. In: Krishnaswami, S., Cochran, J.K. (Eds.), U-Th series nuclides in aquatic systems. Elsevier, Amsterdam, pp. 105-153.

Post, V.E.A., 2006. PHREEQC for Windows [software version 2.13.00]. http://www.geo. vu.nl/users/posv/phreeqc/index.html.

Puigdomenech, I., Trotignon, L., Kotelnikova, S., Pedersen, K., Griffault, L., Michaud, V., Lartigue, J., Hama, K., Yoshida, H., West, J.M., Bateman, K., Milodowski, A.E., Banwart, S.A., Rivas Perez, J., Tullborg, E., 2000. $\mathrm{O}_{2}$ consumption in a granitic environment. In: Smith, R.W., Shoesmith, D.W. (Eds.), Scientific Basis for Nuclear Waste Management XXIII. Materials Research Society, Symposium Proceeedings, vol. 608, pp. 179-184.

Rama, Moore, W.S., 1984. Mechanism of transport of U-Th series radioisotopes from solids into ground water. Geochimica et Cosmochimica Acta 48, 395-399.

Reid, D.F., Key, R.M., Schink, D.R., 1979. Radium, thorium, and actinium extraction from seawater using an improved manganese-oxide-coated fiber. Earth and Planetary Science Letters 43, 223-226.

Reynolds, B.C., Wasserburg, G.J., Baskaran, M., 2003. The transport of U- and Th-series nuclides in sandy confined aquifers. Geochimica et Cosmochimica Acta 67, 1955-1972.

Speer, J.A., 1994. Nature of the Rolesville batholith, North Carolina. In: Stoddard, E.F., Blake, D.E. (Eds.), Geology and Field Trip Guide, Western Flank of the Raleigh Metamorphic Belt, North Carolina. Carolina Geological Society, Durham, NC, pp. 57-62.

Speer, J.A., Hoff, K., 1997. Elemental composition of the Alleghanian granitoid plutons of the southern Appalachians. In: Sinha, A.K., Whalen, J.B., Hogan, J.P. (Eds.), The nature of magmatism in the Southern Appalachian Orogen. Memoir, vol. 191. Geological Society of America, Boulder, CO, pp. 287-308.

Speer, J.A., Solberg, T.N., Becker, S.W.,1981. Petrography of the uranium-bearing minerals of the Liberty Hill pluton, South Carolina: Phase assemblages and migration of uranium in granitoid rocks. Economic Geology 76, 2162-2175.

Stoddard, E.F., Farrar, S.S., Horton, J.W., Butler, J.R., Druhan, R.M., 1991. The eastern Piedmont in North Carolina. In: Horton, J.W., Zullo, V.A. (Eds.), The Geology of the Carolinas. University of Tennessee Press, Knoxville, TN, pp. 79-92.

Sturchio, N.C., Banner, J.L., Binz, C.M., Heraty, L.B., Musgrove, M., 2001. Radium geochemistry of ground waters in Paleozoic carbonate aquifers, midcontinent, USA. Applied Geochemistry 16, 109-122.

Stumm, W., Morgan, J.J., 1996. Aquatic chemistry: Chemical equilibria and rates in natural waters, 3rd ed. John Wiley \& Sons, New York.

Szabo, Z Zapecza, OS, 1987. Relation between natural radionuclide activities and chemical constituents in ground water in the Newark Basin, New Jersey. In: Graves, B. (Ed.), Radon, Radium, and other Radioactivity in Ground Water. Lewis Publishers, Chelsea, MI, pp. 283-308.

Szabo, Z., dePaul, V.T., Kraemer, T.F., Parsa, B., 2005. Occurrence of radium-224, radium226 , and radium-228 in water of the unconfined Kirkwood-Cohansey aquifer system, southern New Jersey. U.S. Geological Survey Scientific Investigations Report 2004-5224.

Tarits, C., Aquilina, L., Ayraud, V., Pauwels, H., Davy, P., Touchard, F., Bour, O., 2006. Oxidoreduction sequence related to flux variations of groundwater from a fractured basement aquifer (Ploemeur area, France). Applied Geochemistry 21, 29-47.

Waite, T.D., Davis, J.A., Payne, T.E., Waychunas, G.A., Xu, N., 1994. Uranium(VI) adsorption to ferrihydrite: application of a surface complexation model. Geochimica et Cosmochimica Acta 58, 5465-5478.

Wanty, R.B., Johnson, S.L., Briggs, P.H., 1991. Radon-222 and its parent radionuclides in groundwater from two study areas in New Jersey and Maryland, U.S.A. Applied Geochemistry 6, 305-318.

Wathen, J.B., 1987. The effect of uranium siting in two-mica granites on uranium concentrations and radon activity in ground water In: Graves, B. (Ed.), Radon, Radium, and other Radioactivity in Ground Water. Lewis Publishers, Chelsea, MI, pp. 31-46.

White, A.F., Bullen, T.D., Vivit, D.V., Schulz, M.S., Clow, D.W., 1999. The role of disseminated calcite in the chemical weathering of granitoid rocks. Geochimica et Cosmochimica Acta 63, 1939-1953.

Wood, W.W., Kraemer, T.F., Shapiro, A., 2004. Radon $\left({ }^{222} \mathrm{Rn}\right)$ in ground water of fractured rocks: a diffusion/ion exchange model. Ground Water 42, 552-567. 